\title{
Prognosis prediction model for patients with breast cancer with bone metastasis: based on a population database
}

\author{
Kun-Chi Hua \\ Department of Orthopedic Oncology, Tianjin Hospital, Tianjin, China. \\ Correspondence to: Kun-Chi Hua. Department of Orthopedic Oncology, Tianjin Hospital, Tianjin, China Email: huakunchi93@163.com.
}

\begin{abstract}
Background: Prognosis prediction for breast cancer bone metastasis (BCBM) patients is related to the development of further treatment options, and the judgment of prognosis is often not determined by one factor, which requires a comprehensive assessment of the patient's condition.

Methods: Through the search of the National Cancer Institute database, BCBM patients registered between January 1, 2010 and December 31, 2015 were selected as research goals. All patients were randomly assigned to the model establishment group and validation group at a ratio of 7:3. Log-rank test and multivariate cox regression analysis are used to evaluate each prognostic factor. Selecting the prognostic factor of log-rank test $\mathrm{P}<0.001$ to plot Kaplan-Meier survival curves and use the model establishment group to draw nomgrams. Concordance index (C-index), Receiver Operating Characteristic (ROC) curve and calibration plot are used for internal and external verification.

Results: A total of 13,773 BCBM patients were included in this article, 9,644 BCBM patients were assigned to the model establishment group and 4,129 BCBM patients were assigned to the validation group. Two nomgrams for predicting OS and BCRS are plotted, with C-index of 0.716 and 0.735 , respectively. The ROC curve and the calibration plot drawn using the model establishment group and validation group confirm the prediction accuracy of the two nomgrams.

Conclusions: The final nomograms obtained satisfactory results after a series of internal and external verifications, verifying the accuracy of their predictions. Other samples are needed in the future for more comprehensive external validation of the model, but at this stage, this model will help physicians and patients to have a more accurate judgment of the prognosis.
\end{abstract}

Keywords: Breast cancer bone metastasis (BCBM); Surveillance; Epidemiology, and End Results (SEER); nomogram

Received: 05 April 2020. Accepted: 16 July 2020; Published: 31 January 2021.

doi: $10.21037 /$ tbcr-20-14

View this article at: http://dx.doi.org/10.21037/tbcr-20-14

\section{Introduction}

In 2018, the global cancer report reported by WHO showed that the incidence of breast cancer is second, and it is also one of the most common types of cancer in women (1). Although there are clear early diagnosis and standard treatment methods for breast cancer, the mortality rate remains high (1). Breast cancer is prone to distant metastasis. The incidence of bone metastases in advanced breast cancer patients is about $70 \%$ (2). The first part of the patients with metastases is bone $(2,3)$. Even breast cancer patients who are reasonably treated have a risk of developing bone metastases (3). Breast cancer bone metastasis (BCBM) often has no obvious symptoms in the early stage, so it is easy to be ignored by patients (4). If the symptoms of bone pain occur, the patient has already entered the late stage of breast cancer (5). The most common manifestations of breast cancer patients with bone metastases are severe pain, pathological fractures, spinal cord compression and other bone-related adverse events $(4,5)$. Because breast cancer patients usually have a long survival time, the existence of these adverse events seriously affects the quality of life of BCBM patients. 
Establishing a prognostic prediction model usually requires a suitable statistical method and a relatively large sample size. By summarizing the basic conditions and treatment of a large number of breast cancer patients, and using reasonable statistical methods to analyze the prognosis related factors, and then establish a simple and efficient prognosis prediction model. The Surveillance, Epidemiology, and End Results (SEER) database administered by the National Cancer Institute contains data on cancer patients from a number of medical centers, providing a large and well-established demographic, tumor pathology, and treatment information for breast cancer patients. It is essential for us to use the big data to establish a reasonable prognostic prediction model (6).

The aim of this study was to collect information on the demographics, tumor pathology, and treatment of patients with breast cancer who were diagnosed with bone metastases in the SEER database. Describe the basic condition and median survival time of BCBM patients. In addition, multivariate cox regression was used to evaluate the impact of each independent factor on prognosis. Finally, cox regression results were visualized by plotting nomograms, and internal and external validation of these nomograms was performed to measure the accuracy of these nomograms for prognosis prediction. We present the following article in accordance with the STROBE reporting checklist (available at http://dx.doi.org/10.21037/tbcr-20-14).

\section{Methods}

\section{Data collection}

The National Cancer Institute's SEER database covers about $28 \%$ of the population of the United States and collects data on cancer patients from 18 tumor registration centers (6). The latest data for the (1973-2016 varying) database released in November 2018 was obtained using SEER stat special software (version 8.3.5), and data acquisition was done in client-server mode (7). During the period from January 1, 2010 to December 31, 2015, a total of 13,773 breast cancer patients were diagnosed with bone metastases. Exclusion criteria include: no/unknown breast cancer patients with bone metastases, unknown survival time and vital status.

\section{Inclusion codes and criteria}

The main end points of the study were overall survival (OS) and breast cancer-related survival (BCRS). In this study, we classified patients according to the following factors, such as age $(\leq 45,46-65,66-85, \leq 86)$, gender (Famale, Male), race (White, Black, Asian or Pacific Islander, Others) and marital status (Married, Unmarried, Unknown).

For the tumor, the tumors were classified according to grade(I, II, III, IV, Unknown), laterality (Left, Right, Other), tumor size ( $\leq 20 \mathrm{~mm}, 21-50 \mathrm{~mm},>50 \mathrm{~mm})$, T stage $(0,1,2,3,4, X), \mathrm{N}$ stage $(0,1,2,3, \mathrm{X})$, histological type (Ductal, Lobular, Adenocarcinoma, Other), subtypes (HR+/ HER2 - (Luminal A), HR+/HER2 + (Luminal B), HR-/ HER2+ (HER2 enriched), HR-/HER2- (Triple Negative), Unknown) and number of extra-bone (brain, liver and lung) metastatic organs $(0,1,2,3$, Unknown). In addition, this study also collected treatments for primary breast cancer lesions, including surgery (Yes, No), chemotherapy (Yes, No) and radiotherapy (Yes, No).

\section{Patients grouping}

In order to establish an effective prognostic prediction model, all patients were divided into a model establishment group and a verification group according to a random assignment method. Among them, the model establishment group included a total of 9,464 patients, and the validation group included 4,129 patients. Both groups of patients will be considered when the final nomograms are drawn.

\section{Statistical analysis}

Basic information about BCBM patients using a method of descriptive statistics. The chi-square test was used to analyze the dead/live of categorical variables of prognostic factors in BCBM patients. The survival time of each prognostic factor is expressed as the median and interquartile ranges. Kaplan-Meier survival curves and log-rank test were used to analyze the OS and BCRS for each prognostic factor. Multivariate cox regression analysis was used to analyze allcause mortality (ACM) and breast cancer-related mortality (BCRM) for each prognostic factor and categorical variable. Moreover, the hazard ratios (HR) and $95 \%$ CIs for all strata of each factor are also calculated. The $\mathrm{P}$ value $<0.05$ is considered statistically significant.

\section{Plotting Kaplan-Meier survival curves and construction of nomograms}

Selecting the prognostic factor of log-rank test $\mathrm{P}<0.001$ 


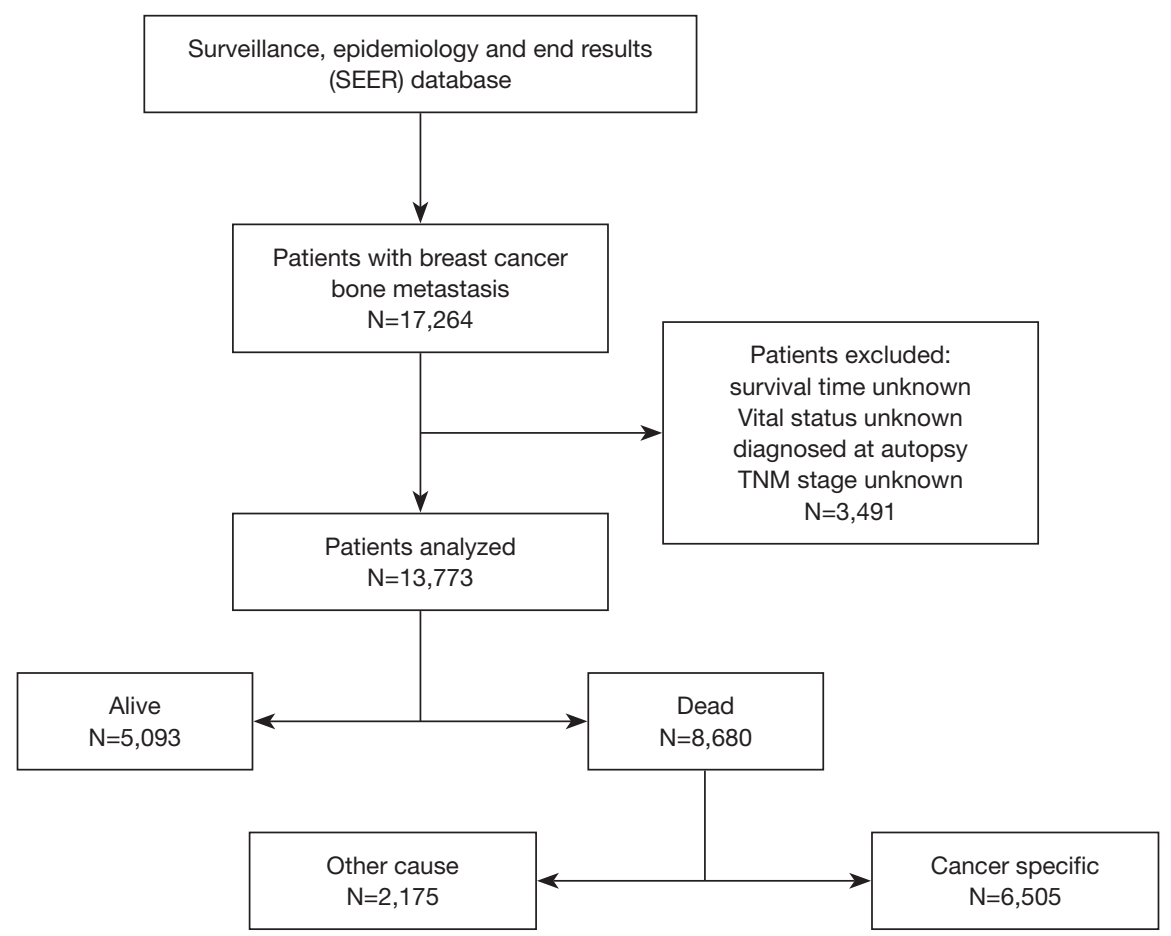

Figure 1 Flowchart of patients identification and selection.

to plot Kaplan-Meier survival curves. Based on the results of multivariate cox regression analysis, the prognostic predictors of $\mathrm{P}<0.001$ in the log-rank test were included in the nomogram. The model was used to model establishment group data for internal verification of the nomograms, and the validation group data was used for external verification of the nomograms. The Concordance index (C-index), Receiver operating characteristic (ROC) curve and calibration curve were used to evaluate the predictive power of the model (8). The C-index is between 0.5 and $1,0.5$ is completely inconsistent, indicating that the model has no predictive effect, and 1 is completely consistent, indicating that the model's prediction results are completely consistent with the actual. In general, the C-index is less accurate at 0.50-0.70: moderate accuracy between 0.71 and 0.90 ; and high accuracy above 0.90 . The area under the ROC curve (AUC) refers to the area around the ROC curve and the $\mathrm{x}$-axis, $(1,0)-(1,1)$. Similar to the C-index, the AUC is less accurate at $0.50-0.70$ : moderate accuracy between 0.71 and 0.90 ; and high accuracy above $0.90(9,10)$. The predicted probability of the nomograms of the OS and BCRS for 1,3 and 5 years are compared with the observed survival probability to obtain calibration plots. All statistical analysis, model establishment group and validation group generation and construction of nomograms were performed by $\mathrm{R}$ project (Version 3.6.0).

\section{Results}

\section{Demographic and tumor pathological features of BCBM patients}

The specific screening process is shown in Figure 1. Between Jan 1, 2010 and Dec 31, 2015, 13,773 BCBM patients were included in this article, 9,644 BCBM patients were assigned to the model establishment group and 4,129 BCBM patients were assigned to the validation group. From 2010 to 2015 , the number of BCBM patients was basically stable. The demographic and tumor pathology information of BCBM was shown in Table 1, and the median survival was shown in Table 2.

The mean age and median age of 13,773 patients were 62.05 and 62 years, respectively. In entire group, the majority of the categorical variables in this study were 46-65 years old $(60.3 \%)$, female $(98.7 \%)$, white $(77.0 \%)$, unmarried $(51.9 \%)$, grade II $(34.7 \%)$, left $(48.3 \%)$, tumor size $21-50 \mathrm{~mm}(35.5 \%)$, T4 (26.7\%), N1 (41.0\%), ductal $(61.6 \%)$, number of extra-bone metastatic organs was 
Page 4 of 21

Table 1 Demographic information and tumor pathology information of BCBM

\begin{tabular}{|c|c|c|c|c|c|c|}
\hline \multirow{2}{*}{ Characteristics } & \multicolumn{2}{|c|}{ Entire Group } & \multicolumn{2}{|c|}{ Model Establishment Group } & \multicolumn{2}{|c|}{ Validation Group } \\
\hline & No & $\%$ & No & $\%$ & No & $\%$ \\
\hline Total & 13,773 & 100.00 & 9,644 & 1.000 & 4,129 & 100.00 \\
\hline \multicolumn{7}{|l|}{ Age at diagnosis } \\
\hline$\leq 45$ & 1,688 & 12.30 & 1,164 & 0.121 & 524 & 12.70 \\
\hline $46-65$ & 6,643 & 48.20 & 4,696 & 0.487 & 1,947 & 47.20 \\
\hline $66-85$ & 4,773 & 34.70 & 3,321 & 0.344 & 1,452 & 35.20 \\
\hline$\geq 86$ & 669 & 4.90 & 463 & 0.048 & 206 & 5.00 \\
\hline \multicolumn{7}{|l|}{ Gender } \\
\hline Female & 13,600 & 98.70 & 9,519 & 0.987 & 4,081 & 98.80 \\
\hline Male & 173 & 1.30 & 125 & 0.013 & 48 & 1.20 \\
\hline \multicolumn{7}{|l|}{ Race } \\
\hline White & 10,612 & 77.00 & 7,425 & 0.770 & 3,187 & 77.20 \\
\hline Black & 2,126 & 15.40 & 1,477 & 0.153 & 649 & 15.70 \\
\hline Asian or Pacific Islander & 911 & 6.60 & 654 & 0.068 & 257 & 6.20 \\
\hline Other & 124 & 0.90 & 88 & 0.009 & 36 & 0.90 \\
\hline \multicolumn{7}{|l|}{ Marital status } \\
\hline Married & 5,876 & 42.70 & 4,107 & 0.426 & 1,769 & 42.80 \\
\hline Unmarried & 7,146 & 51.90 & 5,022 & 0.521 & 2,124 & 51.40 \\
\hline Unknown & 751 & 5.50 & 515 & 0.053 & 236 & 5.70 \\
\hline \multicolumn{7}{|l|}{ Year of diagnosis } \\
\hline 2010 & 2,062 & 15.00 & 1,469 & 0.152 & 593 & 14.40 \\
\hline 2011 & 2,214 & 16.10 & 1,541 & 0.160 & 673 & 16.30 \\
\hline 2012 & 2,229 & 16.20 & 1,560 & 0.162 & 669 & 16.20 \\
\hline 2013 & 2,450 & 17.80 & 1,702 & 0.176 & 748 & 18.10 \\
\hline 2014 & 2,418 & 17.60 & 1,678 & 0.174 & 740 & 17.90 \\
\hline 2015 & 2,400 & 17.40 & 1,694 & 0.176 & 706 & 17.10 \\
\hline \multicolumn{7}{|l|}{ Grade } \\
\hline I & 994 & 7.20 & 699 & 0.072 & 295 & 7.10 \\
\hline ॥ & 4,773 & 34.70 & 3,334 & 0.346 & 1,439 & 34.90 \\
\hline III & 4,256 & 30.90 & 2,956 & 0.307 & 1,300 & 31.50 \\
\hline IV & 64 & 0.50 & 51 & 0.005 & 13 & 0.30 \\
\hline Unknown & 3,686 & 26.80 & 2,604 & 0.270 & 1,082 & 26.20 \\
\hline \multicolumn{7}{|l|}{ Laterality } \\
\hline Left & 6,652 & 48.30 & 4,649 & 0.482 & 2,003 & 48.50 \\
\hline Right & 6,347 & 46.10 & 4,464 & 0.463 & 1,883 & 45.60 \\
\hline Other & 774 & 5.60 & 531 & 0.055 & 243 & 5.90 \\
\hline
\end{tabular}

Table 1 (continued) 
Table 1 (continued)

\begin{tabular}{|c|c|c|c|c|c|c|}
\hline \multirow{2}{*}{ Characteristics } & \multicolumn{2}{|c|}{ Entire Group } & \multicolumn{2}{|c|}{ Model Establishment Group } & \multicolumn{2}{|c|}{ Validation Group } \\
\hline & No & $\%$ & No & $\%$ & No & $\%$ \\
\hline \multicolumn{7}{|l|}{ Tumor size } \\
\hline$\leq 20 \mathrm{~mm}$ & 2,249 & 16.30 & 1,588 & 0.165 & 661 & 16.00 \\
\hline $21-50 \mathrm{~mm}$ & 4,892 & 35.50 & 3,401 & 0.353 & 1,491 & 36.10 \\
\hline$>50 \mathrm{~mm}$ & 3,933 & 28.60 & 2,772 & 0.287 & 1,161 & 28.10 \\
\hline Unknown & 2,699 & 19.60 & 1,883 & 0.195 & 816 & 19.80 \\
\hline \multicolumn{7}{|l|}{ stage_T } \\
\hline T0 & 305 & 2.20 & 212 & 0.022 & 93 & 2.30 \\
\hline $\mathrm{T} 1$ & 1,594 & 11.60 & 1,131 & 0.117 & 463 & 11.20 \\
\hline $\mathrm{T} 2$ & 3,627 & 26.30 & 2,520 & 0.261 & 1,107 & 26.80 \\
\hline T3 & 1,874 & 13.60 & 1,301 & 0.135 & 573 & 13.90 \\
\hline T4 & 3,683 & 26.70 & 2,738 & 0.284 & 945 & 22.90 \\
\hline $\mathrm{TX}$ & 2,510 & 18.20 & 1,742 & 0.181 & 768 & 18.60 \\
\hline \multicolumn{7}{|l|}{ stage_N } \\
\hline No & 3,455 & 25.10 & 2,442 & 0.253 & 1,013 & 24.50 \\
\hline N1 & 5,650 & 41.00 & 3,960 & 0.411 & 1,690 & 40.90 \\
\hline $\mathrm{N} 2$ & 1,321 & 9.60 & 910 & 0.094 & 411 & 10.00 \\
\hline N3 & 1,615 & 11.70 & 1,115 & 0.116 & 500 & 12.10 \\
\hline$N X$ & 1,732 & 12.60 & 1,217 & 0.126 & 515 & 12.50 \\
\hline \multicolumn{7}{|l|}{ Histological type } \\
\hline Ductal & 8,480 & 61.60 & 5,978 & 0.620 & 2,502 & 60.60 \\
\hline Lobular & 1,727 & 12.50 & 1,179 & 0.122 & 548 & 13.30 \\
\hline Adenocarcinoma & 872 & 6.30 & 604 & 0.063 & 268 & 6.50 \\
\hline Other & 2,694 & 19.60 & 1,883 & 0.195 & 811 & 19.60 \\
\hline \multicolumn{7}{|l|}{ Other metastases ${ }^{*}$} \\
\hline 0 & 7,445 & 54.10 & 5,216 & 0.541 & 2,229 & 54.00 \\
\hline 1 & 3,838 & 27.90 & 2,687 & 0.279 & 1,151 & 27.90 \\
\hline 2 & 1,368 & 9.90 & 954 & 0.099 & 414 & 10.00 \\
\hline 3 & 239 & 1.70 & 169 & 0.018 & 70 & 1.70 \\
\hline Unknown & 883 & 6.40 & 618 & 0.064 & 265 & 6.40 \\
\hline \multicolumn{7}{|l|}{ Subtypes } \\
\hline HR+/HER2- (Luminal A) & 7,945 & 57.70 & 5,538 & 0.574 & 2,407 & 58.30 \\
\hline HR+/HER2+ (Luminal B) & 1,844 & 13.40 & 1,315 & 0.136 & 529 & 12.80 \\
\hline HR-/HER2+ (HER2 enriched) & 715 & 5.20 & 488 & 0.051 & 227 & 5.50 \\
\hline HR-/HER2- (Triple negative) & 1,081 & 7.80 & 747 & 0.077 & 334 & 8.10 \\
\hline Unknown & 2,188 & 15.90 & 1,556 & 0.161 & 632 & 15.30 \\
\hline
\end{tabular}

Table 1 (continued) 
Table 1 (continued)

\begin{tabular}{|c|c|c|c|c|c|c|}
\hline \multirow{2}{*}{ Characteristics } & \multicolumn{2}{|c|}{ Entire Group } & \multicolumn{2}{|c|}{ Model Establishment Group } & \multicolumn{2}{|c|}{ Validation Group } \\
\hline & No & $\%$ & No & $\%$ & No & $\%$ \\
\hline \multicolumn{7}{|l|}{ Surgery } \\
\hline Yes & 3,461 & 25.10 & 2,420 & 0.251 & 1,041 & 25.20 \\
\hline No & 10,312 & 74.90 & 7,224 & 0.749 & 3,088 & 74.80 \\
\hline \multicolumn{7}{|l|}{ Chemotherapy } \\
\hline Yes & 6,549 & 47.50 & 4,601 & 0.477 & 1,948 & 47.20 \\
\hline No & 7,224 & 52.50 & 5,043 & 0.523 & 2,181 & 52.80 \\
\hline \multicolumn{7}{|l|}{ Radiotherapy } \\
\hline Yes & 4,688 & 34.00 & 3,320 & 0.344 & 1,368 & 33.10 \\
\hline No & 9,085 & 66.00 & 6,324 & 0.656 & 2,761 & 66.90 \\
\hline
\end{tabular}

*, number of extra-bone (brain, liver and lung) metastatic organs. BCBM, breast cancer bone metastasis.

$0(54.1 \%)$, luminal A $(57.7 \%)$, no surgery $(74.9 \%)$, no chemotherapy (52.5\%), and no radiotherapy $(66.0 \%)$.

In model establishment group, the majority of the categorical variables in this study were 46-65 years old (48.7\%), female (98.7\%), white (77.0\%), unmarried (52.1\%), grade II $(34.6 \%)$, left $(48.2 \%)$, tumor size $21-50 \mathrm{~mm}$ (35.3\%), T4 (28.4\%), N1 (41.1\%), ductal (62.0\%), number of extra-bone metastatic organs was 0 (54.1\%), luminal A (57.4\%), no surgery (74.9\%), no chemotherapy $(52.3 \%)$, and no radiotherapy (65.6\%).

In validation group, the majority of the categorical variables in this study were 46-65 years old (47.2\%), female $(98.8 \%)$, white $(77.2 \%)$, unmarried $(51.4 \%)$, grade II (34.9\%), left (48.5\%), tumor size $21-50 \mathrm{~mm}(36.1 \%)$, T2 (26.8\%), N1 (40.9\%), ductal (60.6\%), number of extrabone metastatic organs was $0(54.0 \%)$, luminal A (58.3\%), no surgery $(74.8 \%)$, no chemotherapy $(52.8 \%)$, and no radiotherapy $(66.9 \%)$.

\section{The impact of different variables on ACM and BCRM}

Among all 13,773 BCBM patients, 8,680 (63.0\%) patients with ACM, while 5,093 (43.9\%) died of breast cancer (Figure 1, Table 3). Observing the demographic data, whether due to ACM or BCRM, with the age at diagnosis increases, the mortality rate also increases significantly $(\mathrm{P}<0.001$ and $\mathrm{P}<0.001)$, however, gender has no significant effect on mortality in patients with breast cancer with bone metastasis $(\mathrm{P}=0.638$ and $\mathrm{P}=0.876)$. Blacks have the highest ACM (69.8\%) and BCRM (63.6\%). Unmarried patients have the highest ACM (68.1\%) and BCRM (61.2\%). The diagnosis year was from 2010 to 2015, and the patient's ACM and BCRM decreased gradually.

Observing tumor pathology data, ACM and BCRM are basically the same between the left and right primary tumors. As the size of the primary tumor increases, ACM and BCRM also show an upward trend. Primary tumor of stage T4 has the highest ACM (68.1\%) and BCRM (61.2\%). Primary tumor of stage NX has the highest ACM (74.8\%) and BCRM (68.4\%), however, ACM and BCRM in N0 to N4 are basically the same. Among the histological types, ACM and BCRM of ductal and lobular carcinoma are basically the same, and both are lower than adenocarcinoma. Patients with extra-bone metastases in the brain, lung and liver have the highest ACM (86.2\%) and BCRM $(83.7 \%)$. In addition, the increase in the number of extra-bone metastatic organs, ACM and BCRM have also increased. Among the subtypes, triple negative breast cancer patients have the highest ACM and BCRM.

Observing treatment data, ACM $(66.7 \%$ vs. $52.2 \%$, $\mathrm{P}<0.001)$ and $\mathrm{BCRM}(60.1 \%$ vs. $44.6 \%, \mathrm{P}<0.001)$ in those patients with primary tumors who were not undergoing surgery were significantly higher than those undergoing surgery. ACM $(69.2 \%$ vs. $56.2 \%, \mathrm{P}<0.001)$ and $\mathrm{BCRM}$ $(62.0 \%$ vs. $50.1 \%, \mathrm{P}<0.001)$ were significantly higher in those who did not receive chemotherapy than those receiving chemotherapy. Similarly, patients who did not receive radiotherapy had significantly higher ACM (64.3\% vs. $60.5 \%, \mathrm{P}<0.001)$ and $\mathrm{BCRM}(56.9 \%$ vs. $54.6 \%, \mathrm{P}=0.016)$ than those receiving radiotherapy. 
Table 2 Median survival and survival months of BCBM patients

\begin{tabular}{|c|c|c|}
\hline Characteristics & $\begin{array}{l}\text { Patients, } \\
\text { No }\end{array}$ & $\begin{array}{c}\text { Median survival } \\
\text { months }\end{array}$ \\
\hline Total & 13,773 & $20.0[7-36]$ \\
\hline \multicolumn{3}{|l|}{ Age at diagnosis } \\
\hline$\leq 45$ & 1,688 & 27.0 [14-43] \\
\hline $46-65$ & 6,643 & 22.0 [10-38] \\
\hline $66-85$ & 4,773 & $17.0[4-32]$ \\
\hline$\geq 86$ & 669 & 8.0 [1-22] \\
\hline \multicolumn{3}{|l|}{ Gender } \\
\hline Female & 13,600 & $20.0[7-36]$ \\
\hline Male & 173 & $18.0[7-33]$ \\
\hline \multicolumn{3}{|l|}{ Race } \\
\hline White & 10,612 & $20.0[7-37]$ \\
\hline Black & 2,126 & $17.0[6-31]$ \\
\hline Asian or Pacific Islander & 911 & $21.0[8-36]$ \\
\hline Other & 124 & $18.0[8-35]$ \\
\hline \multicolumn{3}{|l|}{ Marital status } \\
\hline Married & 5,876 & 23.0 [11-39] \\
\hline Unmarried & 7,146 & $18.0[5-33]$ \\
\hline Unknown & 751 & $20.0[8-34]$ \\
\hline \multicolumn{3}{|l|}{ Grade } \\
\hline I & 994 & $26.0[14-42]$ \\
\hline II & 4,773 & 24.0 [12-40] \\
\hline III & 4,256 & $18.0[7-33]$ \\
\hline IV & 64 & $13.5[5-29]$ \\
\hline Unknown & 3,686 & $16.0[3-32]$ \\
\hline \multicolumn{3}{|l|}{ Laterality } \\
\hline Left & 6,652 & $20.0[7-36]$ \\
\hline Right & 6,347 & $21.0[7-37]$ \\
\hline Other & 774 & $16.0[3-30]$ \\
\hline \multicolumn{3}{|l|}{ Tumor size } \\
\hline$\leq 20 \mathrm{~mm}$ & 2,249 & 23.0 [10-39] \\
\hline $21-50 \mathrm{~mm}$ & 4,892 & $22.0[9-38]$ \\
\hline$>50 \mathrm{~mm}$ & 3,933 & $19.0[7-35]$ \\
\hline Unknown & 2,699 & 15.0 [3-31] \\
\hline \multicolumn{3}{|l|}{ stage_T } \\
\hline TO & 305 & 19.0 [7-35] \\
\hline $\mathrm{T} 1$ & 1,594 & 24.0 [11-40] \\
\hline
\end{tabular}

Table 2 (continued)
Table 2 (continued)

\begin{tabular}{|c|c|c|}
\hline Characteristics & $\begin{array}{l}\text { Patients, } \\
\text { No }\end{array}$ & $\begin{array}{l}\text { Median survival } \\
\text { months }\end{array}$ \\
\hline $\mathrm{T} 2$ & 3,627 & $23.0[12-40]$ \\
\hline T3 & 1,874 & $21.0[11-38]$ \\
\hline $\mathrm{T} 4$ & 3,683 & $18.0[6-33]$ \\
\hline TX & 2,510 & $15.0[2-31]$ \\
\hline \multicolumn{3}{|l|}{ stage_N } \\
\hline NO & 3,455 & $20.0[6-36]$ \\
\hline N1 & 5,650 & $20.0[9-36]$ \\
\hline N2 & 1,321 & 23.0 [12-39] \\
\hline N3 & 1,615 & $21.0[11-38]$ \\
\hline NX & 1,732 & $13.5[2-31]$ \\
\hline \multicolumn{3}{|l|}{ Histological type } \\
\hline Ductal & 8,480 & $21.0[9-38]$ \\
\hline Lobular & 1,727 & $23.0[11-38]$ \\
\hline Adenocarcinoma & 872 & $15.0[3-31]$ \\
\hline Other & 2,694 & $16.0[3-31]$ \\
\hline \multicolumn{3}{|l|}{ Other metastases ${ }^{*}$} \\
\hline 0 & 7,445 & $24.0[13-40]$ \\
\hline 1 & 3,838 & $17.0[5-33]$ \\
\hline 2 & 1,368 & $10.0[2-25]$ \\
\hline 3 & 239 & $4.0[1-16]$ \\
\hline Unknown & 883 & $15.0[3-31]$ \\
\hline \multicolumn{3}{|l|}{ Subtypes } \\
\hline HR+/HER2- (Luminal A) & 7,945 & $23.0[12-38]$ \\
\hline HR+/HER2+ (Luminal B) & 1,844 & $24.0[12-41]$ \\
\hline HR-/HER2+ (HER2 enriched) & 715 & $18.0[6-35]$ \\
\hline HR-/HER2- (Triple negative) & 1,081 & $9.0[3-17]$ \\
\hline Unknown & 2,188 & $10.0[1-28]$ \\
\hline \multicolumn{3}{|l|}{ Surgery } \\
\hline Yes & 3,461 & 29.0 [16-47] \\
\hline No & 10,312 & $17.0[5-32]$ \\
\hline \multicolumn{3}{|l|}{ Chemotherapy } \\
\hline Yes & 6,549 & 22.0 [12-39] \\
\hline No & 7,224 & 17.0 [3-33] \\
\hline \multicolumn{3}{|l|}{ Radiotherapy } \\
\hline Yes & 4,688 & 23.0 [11-40] \\
\hline No & 9,085 & $18.0[5-34]$ \\
\hline
\end{tabular}

*, number of extra-bone (brain, liver and lung) metastatic organs. 
Table 3 Univariate survival analyses of BCBM patients according to various clinicopathological variables Chi-square test)

\begin{tabular}{|c|c|c|c|c|c|c|c|c|c|c|c|c|}
\hline \multirow{3}{*}{ Characteristics } & \multicolumn{6}{|c|}{ All cause } & \multicolumn{6}{|c|}{ Breast cancer-related } \\
\hline & \multirow{2}{*}{ Total } & \multicolumn{2}{|c|}{ Dead } & \multicolumn{2}{|c|}{ Alive } & \multirow{2}{*}{$\mathrm{P}$} & \multirow{2}{*}{ Total } & \multicolumn{2}{|c|}{ Dead } & \multicolumn{2}{|c|}{ Alive } & \multirow{2}{*}{$\mathrm{P}$} \\
\hline & & No & $\%$ & No & $\%$ & & & No & $\%$ & No & $\%$ & \\
\hline$N$ & 13,773 & 8,680 & 0.63 & 5,093 & 0.37 & & 11,598 & 6,505 & 56.10 & 5,093 & 43.90 & \\
\hline Age at diagnosis & & & & & & $<0.001$ & & & & & & $<0.001$ \\
\hline$\leq 45$ & 1,688 & 867 & 0.514 & 821 & 0.486 & & 1,542 & 721 & 46.80 & 821 & 53.20 & \\
\hline $46-65$ & 6,643 & 3,946 & 0.594 & 2,697 & 0.406 & & 5,814 & 3,117 & 53.60 & 2,697 & 46.40 & \\
\hline $66-85$ & 4,773 & 3,307 & 0.693 & 1,466 & 0.307 & & 3,770 & 2,304 & 61.10 & 1,466 & 38.90 & \\
\hline$\geq 86$ & 669 & 560 & 0.837 & 109 & 0.163 & & 472 & 363 & 76.90 & 109 & 23.10 & \\
\hline Gender & & & & & & 0.638 & & & & & & 0.876 \\
\hline Female & 13,600 & 8,568 & 0.63 & 5,032 & 0.37 & & 11,457 & 6,425 & 56.10 & 5,032 & 43.90 & \\
\hline Male & 173 & 112 & 0.647 & 61 & 0.353 & & 141 & 80 & 56.70 & 61 & 43.30 & \\
\hline Race & & & & & & $<0.001$ & & & & & & $<0.001$ \\
\hline White & 10,612 & 6,611 & 0.623 & 4,001 & 0.377 & & 8,930 & 4,929 & 55.20 & 4,001 & 44.80 & \\
\hline Black & 2,126 & 1,485 & 0.698 & 641 & 0.302 & & 1,762 & 1,121 & 63.60 & 641 & 36.40 & \\
\hline Asian or Pacific Islander & 911 & 524 & 0.575 & 387 & 0.425 & & 801 & 414 & 51.70 & 387 & 48.30 & \\
\hline Other & 124 & 60 & 0.484 & 64 & 0.516 & & 105 & 41 & 39.00 & 64 & 61.00 & \\
\hline Marital status & & & & & & $<0.001$ & & & & & & $<0.001$ \\
\hline Married & 5,876 & 3,344 & 0.569 & 2,532 & 0.431 & & 5,084 & 2,552 & 50.20 & 2,532 & 49.80 & \\
\hline Unmarried & 7,146 & 4,865 & 0.681 & 2,281 & 0.319 & & 5,883 & 3,602 & 61.20 & 2,281 & 38.80 & \\
\hline Unknown & 751 & 471 & 0.627 & 280 & 0.373 & & 631 & 351 & 55.60 & 280 & 44.40 & \\
\hline Year of diagnosis & & & & & & $<0.001$ & & & & & & $<0.001$ \\
\hline 2010 & 2,062 & 1,684 & 0.817 & 378 & 0.183 & & 1,629 & 1,251 & 76.80 & 378 & 23.20 & \\
\hline 2011 & 2,214 & 1,749 & 0.79 & 465 & 0.21 & & 1,802 & 1,337 & 74.20 & 465 & 25.80 & \\
\hline 2012 & 2,229 & 1,585 & 0.711 & 644 & 0.289 & & 1,839 & 1,195 & 65.00 & 644 & 35.00 & \\
\hline 2013 & 2,450 & 1,554 & 0.634 & 896 & 0.366 & & 2,076 & 1,180 & 56.80 & 896 & 43.20 & \\
\hline 2014 & 2,418 & 1,255 & 0.519 & 1,163 & 0.481 & & 2,059 & 896 & 43.50 & 1,163 & 56.50 & \\
\hline 2015 & 2,400 & 853 & 0.355 & 1,547 & 0.645 & & 2,193 & 646 & 29.50 & 1,547 & 70.50 & \\
\hline Grade & & & & & & $<0.001$ & & & & & & $<0.001$ \\
\hline I & 994 & 492 & 0.495 & 502 & 0.505 & & 835 & 333 & 39.90 & 502 & 60.10 & \\
\hline II & 4,773 & 2,682 & 0.562 & 2,091 & 0.438 & & 4,061 & 1,970 & 48.50 & 2,091 & 51.50 & \\
\hline III & 4,256 & 2,861 & 0.672 & 1,395 & 0.328 & & 3,643 & 2,248 & 61.70 & 1,395 & 38.30 & \\
\hline IV & 64 & 58 & 0.906 & 6 & 0.094 & & 50 & 44 & 88.00 & 6 & 12.00 & \\
\hline Unknown & 3,686 & 2,587 & 0.702 & 1,099 & 0.298 & & 3,009 & 1,910 & 63.50 & 1,099 & 36.50 & \\
\hline Laterality & & & & & & $<0.001$ & & & & & & $<0.001$ \\
\hline Left & 6,652 & 4,152 & 0.624 & 2,500 & 0.376 & & 5,634 & 3,134 & 55.60 & 2,500 & 44.40 & \\
\hline
\end{tabular}

Table 3 (continued) 
Table 3 (continued)

\begin{tabular}{|c|c|c|c|c|c|c|c|c|c|c|c|c|}
\hline \multirow{3}{*}{ Characteristics } & \multicolumn{6}{|c|}{ All cause } & \multicolumn{6}{|c|}{ Breast cancer-related } \\
\hline & \multirow{2}{*}{ Total } & \multicolumn{2}{|c|}{ Dead } & \multicolumn{2}{|c|}{ Alive } & \multirow{2}{*}{$P$} & \multirow{2}{*}{ Total } & \multicolumn{2}{|c|}{ Dead } & \multicolumn{2}{|c|}{ Alive } & \multirow{2}{*}{$P$} \\
\hline & & No & $\%$ & No & $\%$ & & & No & $\%$ & No & $\%$ & \\
\hline Right & 6,347 & 3,981 & 0.627 & 2,366 & 0.373 & & 5,339 & 2,973 & 55.70 & 2,366 & 44.30 & \\
\hline Other & 774 & 547 & 0.707 & 227 & 0.293 & & 625 & 398 & 63.70 & 227 & 36.30 & \\
\hline Tumor size & & & & & & $<0.001$ & & & & & & $<0.001$ \\
\hline$\leq 20 \mathrm{~mm}$ & 2,249 & 1,289 & 0.573 & 960 & 0.427 & & 1,795 & 835 & 46.50 & 960 & 53.50 & \\
\hline $21-50 \mathrm{~mm}$ & 4,892 & 2,858 & 0.584 & 2,034 & 0.416 & & 4,155 & 2,121 & 51.00 & 2,034 & 49.00 & \\
\hline$>50 \mathrm{~mm}$ & 3,933 & 2,589 & 0.658 & 1,344 & 0.342 & & 3,448 & 2,104 & 61.00 & 1,344 & 39.00 & \\
\hline Unknown & 2,699 & 1,944 & 0.72 & 755 & 0.28 & & 2,200 & 1,445 & 65.70 & 755 & 34.30 & \\
\hline stage_T & & & & & & $<0.001$ & & & & & & $<0.001$ \\
\hline T0 & 305 & 197 & 0.646 & 108 & 0.354 & & 238 & 130 & 54.60 & 108 & 45.40 & \\
\hline $\mathrm{T} 1$ & 1,594 & 859 & 0.539 & 735 & 0.461 & & 1,271 & 536 & 42.20 & 735 & 57.80 & \\
\hline $\mathrm{T} 2$ & 3,627 & 2,030 & 0.56 & 1,597 & 0.44 & & 3,091 & 1,494 & 48.30 & 1,597 & 51.70 & \\
\hline T3 & 1,874 & 1,140 & 0.608 & 734 & 0.392 & & 1,642 & 908 & 27.20 & 734 & 44.70 & \\
\hline $\mathrm{T} 4$ & 3,863 & 2,654 & 0.687 & 1,209 & 0.313 & & 3,341 & 2,132 & 63.80 & 1,209 & 36.20 & \\
\hline $\mathrm{TX}$ & 2,510 & 1,800 & 0.717 & 710 & 0.283 & & 2,015 & 1,305 & 64.80 & 710 & 35.20 & \\
\hline stage_N & & & & & & $<0.001$ & & & & & & $<0.001$ \\
\hline No & 3,455 & 2,132 & 0.617 & 1,323 & 0.383 & & 2,767 & 1,444 & 52.20 & 1,323 & 47.80 & \\
\hline N1 & 5,650 & 3,433 & 0.608 & 2,217 & 0.392 & & 4,912 & 2,695 & 54.90 & 2,217 & 45.10 & \\
\hline N2 & 1,321 & 797 & 0.603 & 524 & 0.397 & & 1,152 & 628 & 54.50 & 524 & 45.50 & \\
\hline N3 & 1,615 & 1,023 & 0.633 & 592 & 0.367 & & 1,382 & 790 & 57.20 & 592 & 42.80 & \\
\hline$N X$ & 1,732 & 1,295 & 0.748 & 437 & 0.252 & & 1,385 & 948 & 68.40 & 437 & 31.60 & \\
\hline Histological type & & & & & & $<0.001$ & & & & & & $<0.001$ \\
\hline Ductal & 8,480 & 5,129 & 0.605 & 3,351 & 0.395 & & 7,241 & 3,890 & 53.70 & 3,351 & 46.30 & \\
\hline Lobular & 1,727 & 1,067 & 0.618 & 660 & 0.382 & & 1,425 & 765 & 53.70 & 660 & 46.30 & \\
\hline Adenocarcinoma & 872 & 603 & 0.692 & 269 & 0.308 & & 723 & 454 & 62.80 & 269 & 37.20 & \\
\hline Other & 2,694 & 1,881 & 0.698 & 813 & 0.302 & & 2,209 & 1,396 & 63.20 & 813 & 36.80 & \\
\hline Other metastases* & & & & & & $<0.001$ & & & & & & $<0.001$ \\
\hline 0 & 7,445 & 4,058 & 0.545 & 3,387 & 0.455 & & 6,311 & 2,924 & 46.30 & 3,387 & 53.70 & \\
\hline 1 & 3,838 & 2,661 & 0.693 & 1,177 & 0.307 & & 3,222 & 2,045 & 63.50 & 1,177 & 36.50 & \\
\hline 2 & 1,368 & 1,088 & 0.795 & 280 & 0.205 & & 1,149 & 869 & 75.60 & 280 & 24.40 & \\
\hline 3 & 239 & 206 & 0.862 & 33 & 0.138 & & 202 & 169 & 83.70 & 33 & 16.30 & \\
\hline Unknown & 883 & 667 & 0.755 & 216 & 0.245 & & 714 & 498 & 69.70 & 216 & 30.30 & \\
\hline Subtypes & & & & & & $<0.001$ & & & & & & $<0.001$ \\
\hline HR+/HER2- (Luminal A) & 7,945 & 4,708 & 0.593 & 3,237 & 0.407 & & 6,714 & 3,477 & 51.80 & 3,237 & 48.20 & \\
\hline
\end{tabular}

Table 3 (continued) 
Table 3 (continued)

\begin{tabular}{|c|c|c|c|c|c|c|c|c|c|c|c|c|}
\hline \multirow{3}{*}{ Characteristics } & \multicolumn{6}{|c|}{ All cause } & \multicolumn{6}{|c|}{ Breast cancer-related } \\
\hline & \multirow{2}{*}{ Total } & \multicolumn{2}{|c|}{ Dead } & \multicolumn{2}{|c|}{ Alive } & \multirow{2}{*}{$\mathrm{P}$} & \multirow{2}{*}{ Total } & \multicolumn{2}{|c|}{ Dead } & \multicolumn{2}{|c|}{ Alive } & \multirow{2}{*}{$\mathrm{P}$} \\
\hline & & No & $\%$ & No & $\%$ & & & No & $\%$ & No & $\%$ & \\
\hline HR+/HER2+ (Luminal B) & 1,844 & 928 & 0.503 & 916 & 0.497 & & 1,668 & 752 & 45.10 & 916 & 54.90 & \\
\hline $\begin{array}{l}\text { HR-/HER2+ (HER2 } \\
\text { enriched) }\end{array}$ & 715 & 426 & 0.596 & 289 & 0.404 & & 632 & 343 & 54.30 & 289 & 45.70 & \\
\hline $\begin{array}{l}\text { HR-/HER2- (Triple } \\
\text { negative) }\end{array}$ & 1,081 & 937 & 0.867 & 144 & 0.133 & & 856 & 712 & 83.20 & 144 & 16.80 & \\
\hline Unknown & 2,188 & 1,681 & 0.768 & 507 & 0.232 & & 1,728 & 1,221 & 70.70 & 507 & 29.30 & \\
\hline Surgery & & & & & & $<0.001$ & & & & & & $<0.001$ \\
\hline Yes & 3,461 & 1,807 & 0.522 & 1,654 & 0.478 & & 2,986 & 1,332 & 44.60 & 1,654 & 55.40 & \\
\hline No & 10,312 & 6,873 & 0.667 & 3,439 & 0.333 & & 8,612 & 5,173 & 60.10 & 3,439 & 39.90 & \\
\hline Chemotherapy & & & & & & $<0.001$ & & & & & & $<0.001$ \\
\hline Yes & 6,549 & 3,678 & 0.562 & 2,871 & 0.438 & & 5,754 & 2,883 & 50.10 & 2,871 & 49.90 & \\
\hline No & 7,224 & 5,002 & 0.692 & 2,222 & 0.308 & & 5,844 & 3,622 & 62.00 & 2,222 & 38.00 & \\
\hline Radiotherapy & & & & & & $<0.001$ & & & & & & 0.016 \\
\hline Yes & 4,688 & 2,835 & 0.605 & 1,853 & 0.395 & & 4,080 & 2,227 & 54.60 & 1,853 & 45.40 & \\
\hline No & 9,085 & 5,845 & 0.643 & 3,240 & 0.357 & & 7,518 & 4,278 & 56.90 & 3,240 & 43.10 & \\
\hline
\end{tabular}

*, number of extra-bone (brain, liver and lung) metastatic organs.

We plotted Kaplan-Meier survival curves for age, grade, subtype, histological type, number of extra-bone metastatic organs, surgery, radiotherapy, and chemotherapy, based on OS and BCRS for BCBM patients (Figure 2). In addition, log-rank test for all variables is shown in Table 4. It is observed from the figure that the increase in age is significantly related to the worsening prognosis (Figure $2 \mathrm{~A}$, Figure $2 B$ ). The primary tumor has a low degree of differentiation, and the high degree of malignancy is significantly associated with poor prognosis (Figure $2 C$, Figure 2D). Observing the relationship between tumor subtype and prognosis, triple-negative breast cancer is significantly associated with poor prognosis (Figure $2 E$, Figure $2 F$ ). Observing the relationship between histological type and prognosis, the prognosis of ductal carcinoma and lobular carcinoma is significantly better than adenocarcinoma and other types (Figure 2G, Figure 2H). The increase in the number of extra-bone metastatic organs is significantly associated with poor prognosis (Figure 2I, Figure 27). Observing the relationship between treatment and prognosis, no surgery at the primary site is significantly associated with poor prognosis (Figure $2 K$, Figure $2 L$ ). Patients who did not receive radiotherapy or chemotherapy were significantly associated with poor prognosis (radiotherapy: Figure 2M, Figure 2N; chemotherapy: Figure 2O, Figure 2P).

\section{Multivariate Cox regression of prognostic factors in BCBM patients and the construction of nomogram}

Multivariate Cox regression analysis of all variables, and hazard ratios (HR) and 95\% CIs are shown in Table 4. In the final established OS and BCRS prognostic prediction models, variables such as age, grade, subtypes, histological type, number of extra-bone metastatic organs, surgery, radiotherapy, and chemotherapy were included. After that, the nomograms were constructed using the prognosis to predict the risk results (Figures 3,4).

\section{Interior and external verification of nomogram}

The multivariate cox regression model was used to generate 1,3 , and 5 years of nomograms for OS and BCRS. In the model establishment group, the C-index of nomgrams of OS and BCRS is 0.716 and 0.726 , respectively. In the 
A

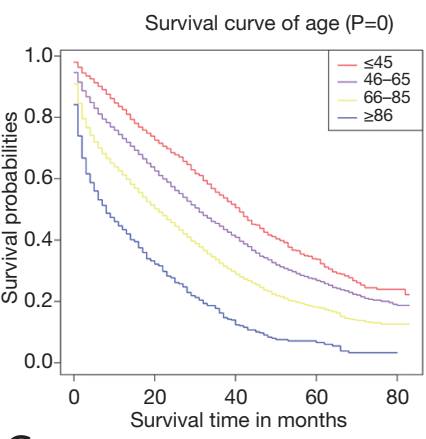

C

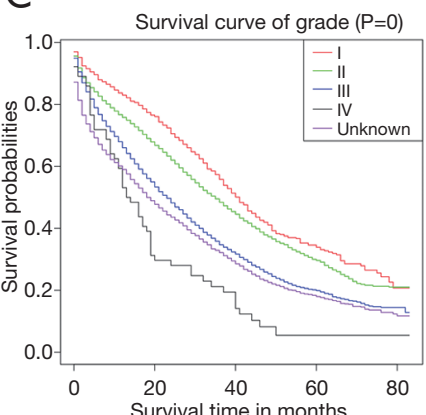

E

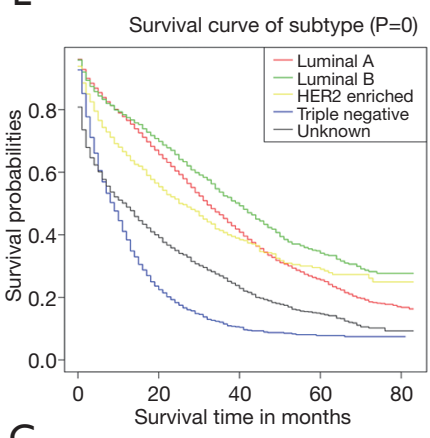

G

Survival curve of histological_type $(P=0)$

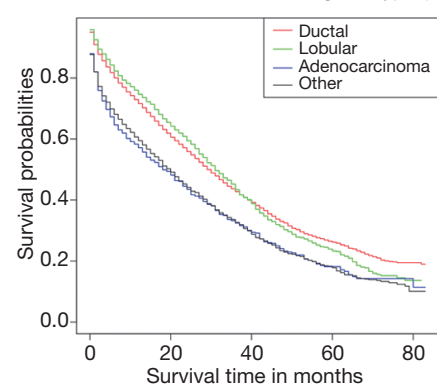

B

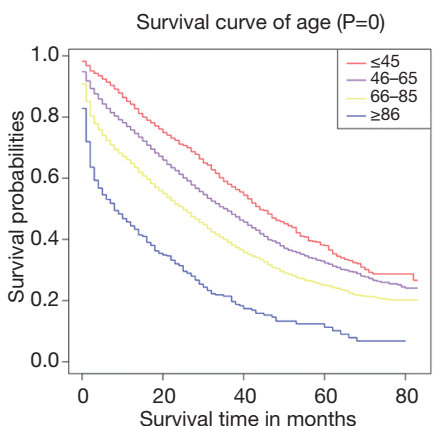

Survival curve of grade $(P=0)$

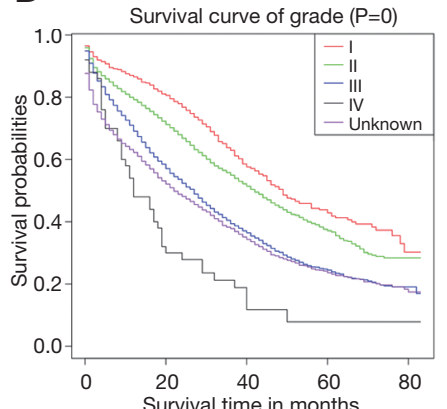

$\mathrm{F}$

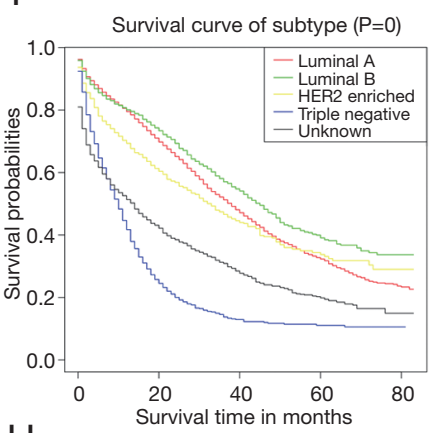

$\mathrm{H}$

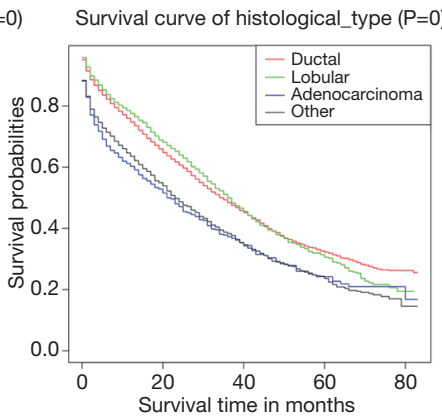

I

Survival curve of other_metastases $(P=0)$

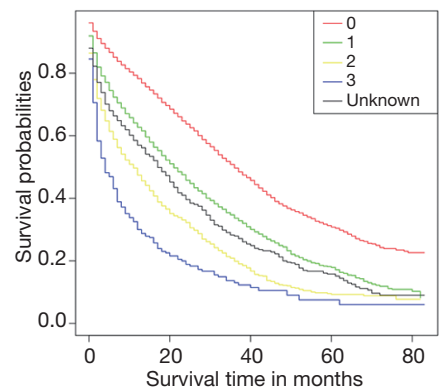

$\mathrm{K}$ Survival curve of surgery $(\mathrm{P}=0)$

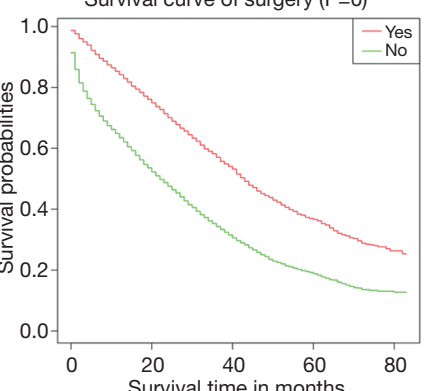

M

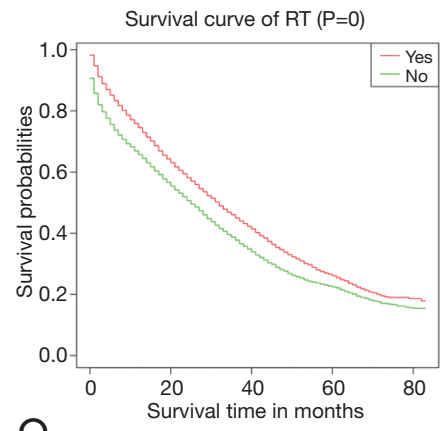

$\mathrm{O}$

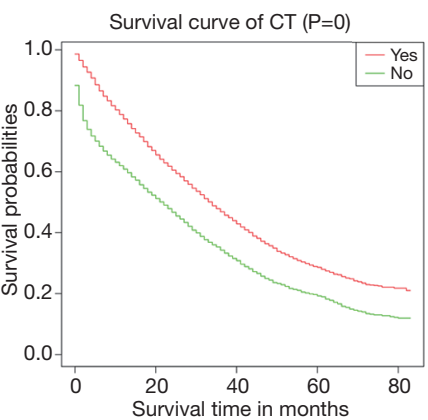

J

Survival curve of other_metastases $(\mathrm{P}=0)$

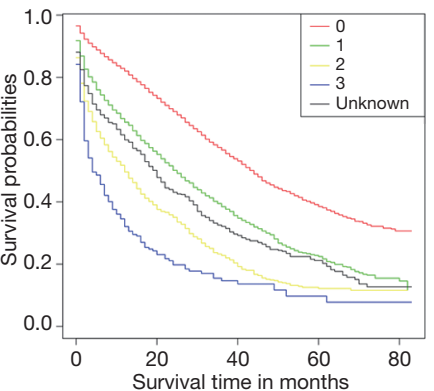

L

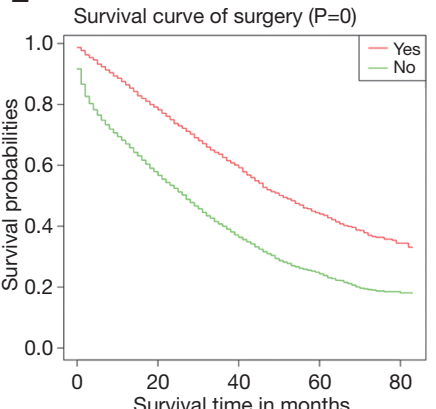

$\mathrm{N}$

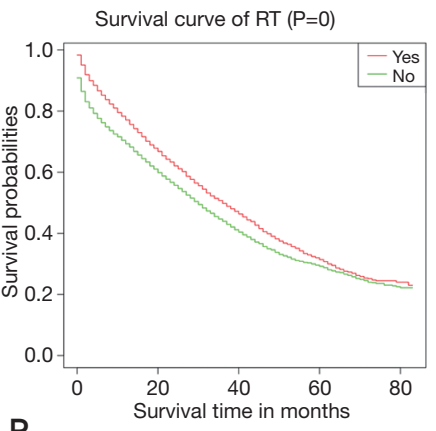

$\mathrm{P}$

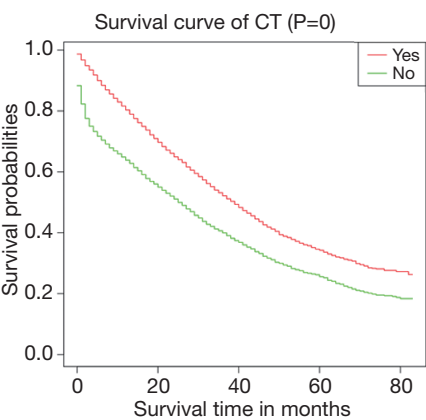

Figure 2 Survival curves in BCBM patients according to different factors. Kaplan-Meier curves among patients stratified by age at diagnosis for OS (A) and BCRS (B); Kaplan-Meier curves among patients stratified by grade for OS (C) and BCRS (D); Kaplan-Meier curves among patients stratified by subtype for OS (E) and BCRS (F). Kaplan-Meier curves among patients stratified by histological type for OS (G) and BCRS (H); Kaplan-Meier curves among patients stratified by other metastases for OS (I) and BCRS (J); Kaplan-Meier curves among patients stratified by surgery/No surgery for OS (K) and BCRS (L); Kaplan-Meier curves among patients stratified by RT/No RT for OS $(\mathrm{M})$ and BCRS $(\mathrm{N})$; Kaplan-Meier curves among patients stratified by CT/No CT for OS (O) and BCRS (P). BCBM, breast cancer bone metastasis; OS, overall survival; BCRS, breast cancer-related survival; CT, chemotherapy; RT, radiotherapy. 
Table 4 Multivariate Cox regression analysis for ACM and BCRM in BCBM patients

\begin{tabular}{|c|c|c|c|c|c|c|c|c|}
\hline \multirow[b]{2}{*}{ Characteristics } & \multicolumn{4}{|c|}{$\mathrm{ACM}$} & \multicolumn{4}{|c|}{ BCRM } \\
\hline & $\mathrm{HR}$ & $95 \% \mathrm{Cl}$ & $P$ value & $\begin{array}{l}\text { Log-rank } \\
\text { (P value) }\end{array}$ & $\mathrm{HR}$ & $95 \% \mathrm{Cl}$ & $P$ value & $\begin{array}{l}\text { Log-rank } \\
\text { (P value) }\end{array}$ \\
\hline Age at diagnosis & & & & $<0.001$ & & & & $<0.001$ \\
\hline$\leq 45$ & 1.000 [reference] & & & & 1.000 [reference] & & & \\
\hline $46-65$ & 1.207 & $1.121-1.300$ & $<0.001$ & & 1.181 & $1.088-1.282$ & $<0.001$ & \\
\hline $66-85$ & 1.558 & $1.441-1.684$ & $<0.001$ & & 1.454 & $1.332-1.587$ & $<0.001$ & \\
\hline$\geq 86$ & 2.324 & $2.076-2.600$ & $<0.001$ & & 2.265 & $1.983-2.588$ & $<0.001$ & \\
\hline Gender & & & & 0.318 & & & & 0.457 \\
\hline Female & 1.000 [reference] & & & & 1.000 [reference] & & & \\
\hline Male & 1.099 & $0.911-1.326$ & 0.324 & & 1.028 & $0.823-1.286$ & 0.809 & \\
\hline Race & & & & 0.003 & & & & 0.002 \\
\hline White & 1.000 [reference] & & & & 1.000 [reference] & & & \\
\hline Black & 1.208 & $1.140-1.281$ & $<0.001$ & & 1.220 & $1.141-1.304$ & $<0.001$ & \\
\hline Asian or Pacific Islander & 0.979 & $0.895-1.071$ & 0.648 & & 0.965 & $0.872-1.068$ & 0.489 & \\
\hline Other & 0.793 & $0.615-1.023$ & 0.075 & & 0.685 & $0.504-0.933$ & 0.016 & \\
\hline Marital status & & & & 0.019 & & & & 0.013 \\
\hline Married & 1.000 [reference] & & & & 1.000 [reference] & & & \\
\hline Unmarried & 1.224 & $1.169-1.281$ & $<0.001$ & & 1.213 & $1.150-1.278$ & $<0.001$ & \\
\hline Unknown & 1.056 & $0.958-1.164$ & 0.274 & & 1.051 & $0.939-1.176$ & 0.39 & \\
\hline Grade & & & & $<0.001$ & & & & $<0.001$ \\
\hline I & 1.000 [reference] & & & & 1.000 [reference] & & & \\
\hline II & 1.195 & $1.085-1.317$ & $<0.001$ & & 1.258 & $1.119-1.415$ & $<0.001$ & \\
\hline III & 1.663 & $1.504-1.839$ & $<0.001$ & & 1.838 & $1.629-2.074$ & $<0.001$ & \\
\hline IV & 2.083 & $1.581-2.745$ & $<0.001$ & & 2.480 & $1.802-3.412$ & $<0.001$ & \\
\hline Unknown & 1.408 & $1.271-1.560$ & $<0.001$ & & 1.509 & $1.334-1.706$ & $<0.001$ & \\
\hline Laterality & & & & 0.008 & & & & 0.004 \\
\hline Left & 1.000 [reference] & & & & 1.000 [reference] & & & \\
\hline Right & 0.967 & $0.926-1.010$ & 0.129 & & 0.970 & $0.922-1.020$ & 0.231 & \\
\hline Other & 0.819 & $0.735-0.912$ & $<0.001$ & & 0.866 & $0.764-0.982$ & 0.025 & \\
\hline Tumor size & & & & 0.002 & & & & $<0.001$ \\
\hline$<20 \mathrm{~mm}$ & 1.000 [reference] & & & & 1.000 [reference] & & & \\
\hline $20-50 \mathrm{~mm}$ & 0.992 & $0.867-1.136$ & 0.910 & & 1.014 & $0.866-1.188$ & 0.86 & \\
\hline$>50 \mathrm{~mm}$ & 1.144 & $1.008-1.300$ & 0.038 & & 1.225 & $1.056-1.420$ & 0.007 & \\
\hline Unknown & 1.061 & $0.932-1.208$ & 0.372 & & 1.136 & $0.975-1.323$ & 0.101 & \\
\hline stage_T & & & & 0.005 & & & & 0.002 \\
\hline TO & 1.000 [reference] & & & & 1.000 [reference] & & & \\
\hline $\mathrm{T} 1$ & 0.98 & $0.825-1.163$ & 0.813 & & 0.977 & $0.791-1.206$ & 0.826 & \\
\hline
\end{tabular}

Table 4 (continued) 
Table 4 (continued)

\begin{tabular}{|c|c|c|c|c|c|c|c|c|}
\hline \multirow[b]{2}{*}{ Characteristics } & \multicolumn{4}{|c|}{$\mathrm{ACM}$} & \multicolumn{4}{|c|}{ BCRM } \\
\hline & $\mathrm{HR}$ & $95 \% \mathrm{Cl}$ & $P$ value & $\begin{array}{c}\text { Log-rank } \\
\text { (P value) }\end{array}$ & $\mathrm{HR}$ & $95 \% \mathrm{Cl}$ & $P$ value & $\begin{array}{l}\text { Log-rank } \\
\text { (P value) }\end{array}$ \\
\hline T2 & 1.074 & $0.868-1.329$ & 0.513 & & 1.156 & $0.896-1.492$ & 0.264 & \\
\hline T3 & 1.043 & $0.844-1.288$ & 0.697 & & 1.118 & $0.869-1.438$ & 0.384 & \\
\hline $\mathrm{T} 4$ & 1.197 & $0.979-1.463$ & 0.08 & & 1.305 & $1.026-1.661$ & 0.03 & \\
\hline $\mathrm{TX}$ & 1.204 & $0.986-1.469$ & 0.068 & & 1.249 & $0.982-1.588$ & 0.07 & \\
\hline stage_N & & & & 0.016 & & & & 0.007 \\
\hline No & 1.000 [reference] & & & & 1.000 [reference] & & & \\
\hline N1 & 0.956 & $0.904-1.011$ & 0.116 & & 1.008 & $0.944-1.077$ & 0.809 & \\
\hline N2 & 1.018 & $0.934-1.108$ & 0.691 & & 1.079 & $0.978-1.190$ & 0.13 & \\
\hline N3 & 1.052 & $0.972-1.138$ & 0.208 & & 1.085 & $0.991-1.189$ & 0.079 & \\
\hline NX & 1.068 & $0.991-1.150$ & 0.083 & & 1.13 & $1.035-1.234$ & 0.006 & \\
\hline Histological type & & & & $<0.001$ & & & & $<0.001$ \\
\hline Ductal & 1.000 [reference] & & & & 1.000 [reference] & & & \\
\hline Lobular & 1.095 & $1.021-1.174$ & 0.011 & & 1.121 & $1.033-1.218$ & 0.007 & \\
\hline Adenocarcinoma & 1.025 & $0.933-1.127$ & 0.603 & & 1.040 & $0.932-1.160$ & 0.481 & \\
\hline Other & 1.194 & $1.126-1.265$ & $<0.001$ & & 1.206 & $1.128-1.291$ & $<0.001$ & \\
\hline Other metastases* & & & & $<0.001$ & & & & $<0.001$ \\
\hline 0 & 1.000 [reference] & & & & 1.000 [reference] & & & \\
\hline 1 & 1.573 & $1.495-1.655$ & $<0.001$ & & 1.658 & $1.564-1.758$ & $<0.001$ & \\
\hline 2 & 2.414 & $2.251-2.589$ & $<0.001$ & & 2.599 & $2.401-2.815$ & $<0.001$ & \\
\hline 3 & 3.521 & $3.050-4.064$ & $<0.001$ & & 3.783 & $3.226-4.437$ & $<0.001$ & \\
\hline Unknown & 1.500 & $1.379-1.632$ & $<0.001$ & & 1.610 & $1.461-1.775$ & $<0.001$ & \\
\hline Subtypes & & & & $<0.001$ & & & & $<0.001$ \\
\hline HR+/HER2- (Luminal A) & 1.000 [reference] & & & & 1.000 [reference] & & & \\
\hline HR+/HER2+ (Luminal B) & 0.856 & $0.795-0.922$ & $<0.001$ & & 0.856 & $0.788-0.930$ & $<0.001$ & \\
\hline HR-/HER2+ (HER2 enriched) & 1.144 & $1.031-1.270$ & 0.011 & & 1.109 & $0.987-1.247$ & 0.083 & \\
\hline HR-/HER2- (Triple negative) & 2.745 & $2.542-2.963$ & $<0.001$ & & 2.858 & $2.616-3.121$ & $<0.001$ & \\
\hline Unknown & 1.519 & $1.428-1.615$ & $<0.001$ & & 1.568 & $1.459-1.685$ & $<0.001$ & \\
\hline Surgery & & & & $<0.001$ & & & & $<0.001$ \\
\hline Yes & 1.000 [reference] & & & & 1.000 [reference] & & & \\
\hline No & 1.529 & $1.444-1.620$ & $<0.001$ & & 1.571 & $1.469-1.679$ & $<0.001$ & \\
\hline Chemotherapy & & & & $<0.001$ & & & & $<0.001$ \\
\hline Yes & 1.000 [reference] & & & & 1.000 [reference] & & & \\
\hline No & 1.540 & $1.465-1.618$ & $<0.001$ & & 1.601 & $1.513-1.695$ & $<0.001$ & \\
\hline Radiotherapy & & & & $<0.001$ & & & & $<0.001$ \\
\hline Yes & 1.000 [reference] & & & & 1.000 [reference] & & & \\
\hline No & 1.095 & $1.045-1.146$ & $<0.001$ & & 1.057 & $1.003-1.114$ & 0.039 & \\
\hline
\end{tabular}

*, number of extra-bone (brain, liver and lung) metastatic organs. ACM, analyze all-cause mortality; BCRM, breast cancer-related mortality. 


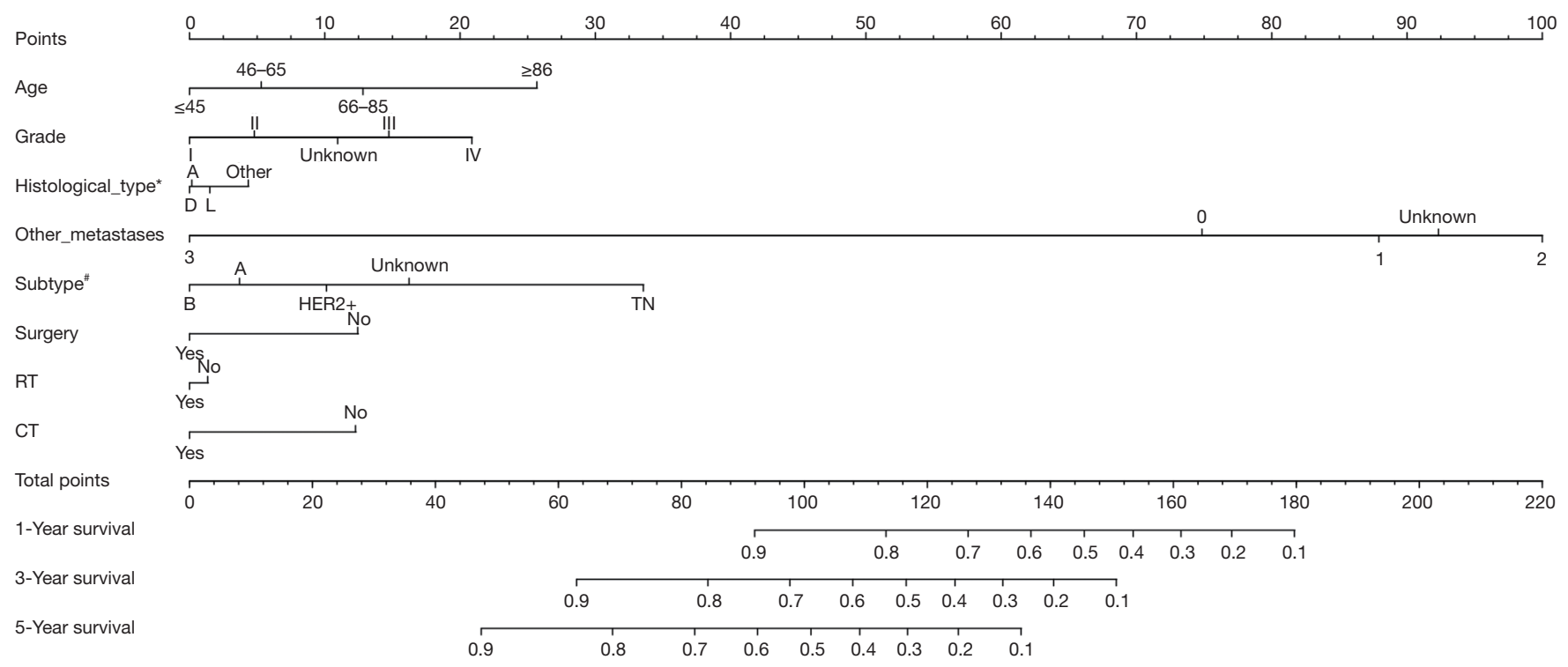

Figure 3 Nomogram of overall survival at 1, 3, and 5 years in patients with breast cancer bone metastasis prediction. *, A, adenocarcinoma; D, ductal carcinoma; L, lobular carcinoma; ", A, luminal A; B, luminal B; HER2+, HER2 enriched; TN, triple negative.

Subints
Surgery
RT
CT

Figure 4 Nomogram of breast cancer-related survival at 1, 3, and 5 years in patients with breast cancer bone metastasis prediction. * A, adenocarcinoma; D, ductal carcinoma; L, lobular carcinoma; * , A, luminal A; B, luminal B; HER2+, HER2 enriched; TN, triple negative.

validation group, the $\mathrm{C}$-index of nomogram of $\mathrm{OS}$ and BCRS is 0.716 and 0.735 , respectively. The ROC curve results of the model establishment group and the validation group are shown in Figures 5 and Figure 6, respectively. The calibration plots of the model establishment group and the validation group show a good consistency between the predicted nomograms of OS and BCRS (Figures 7,8).

\section{Discussion}

The incidence of bone metastasis in breast cancer is high (11). Individualized comprehensive treatment plans should be developed according to the specific conditions to reduce or avoid bone-related events, prolong the survival of patients and improve the quality of life (12-14). The 
A

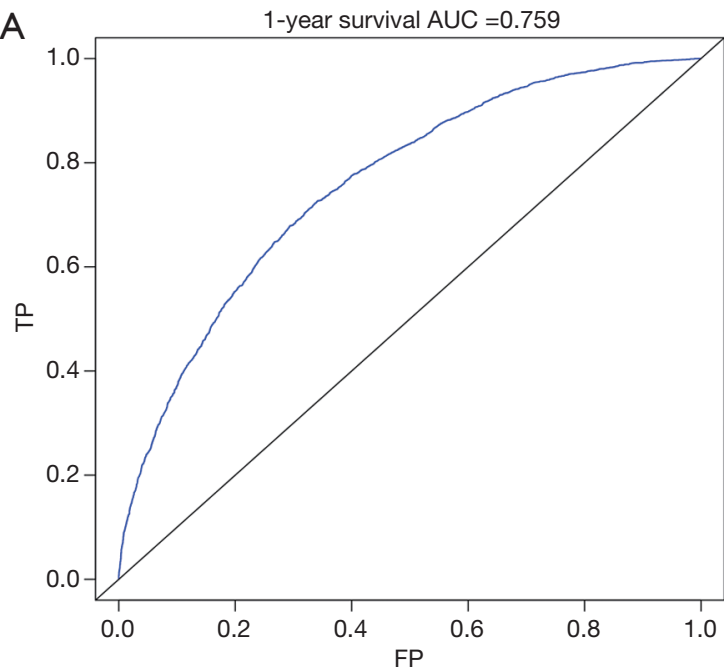

C

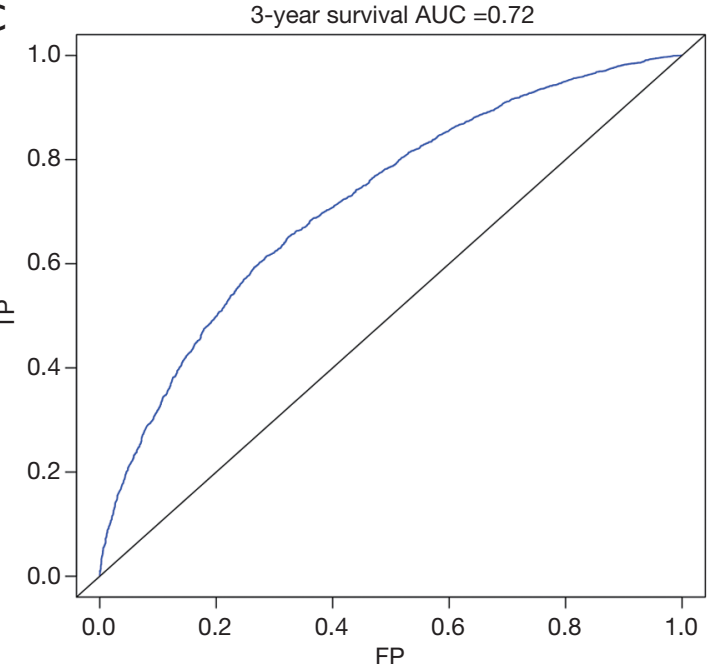

$\mathrm{E}$

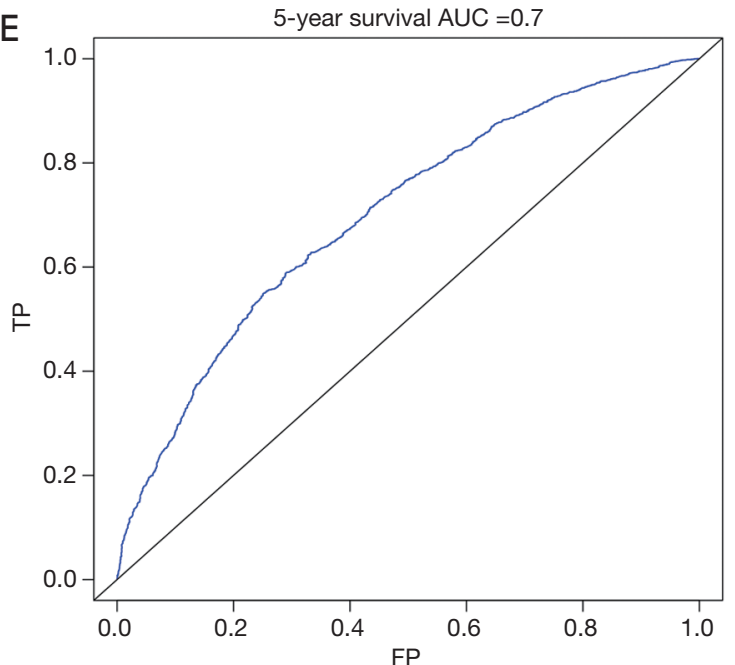

B

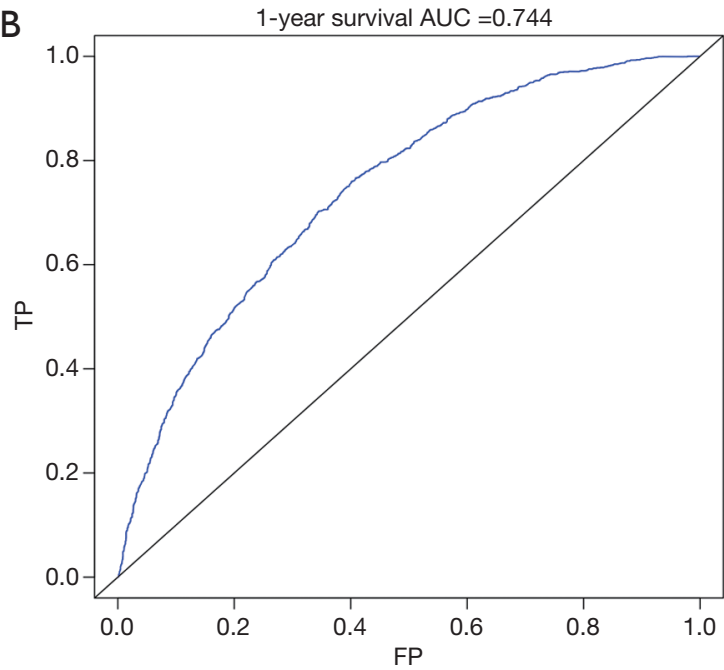

D

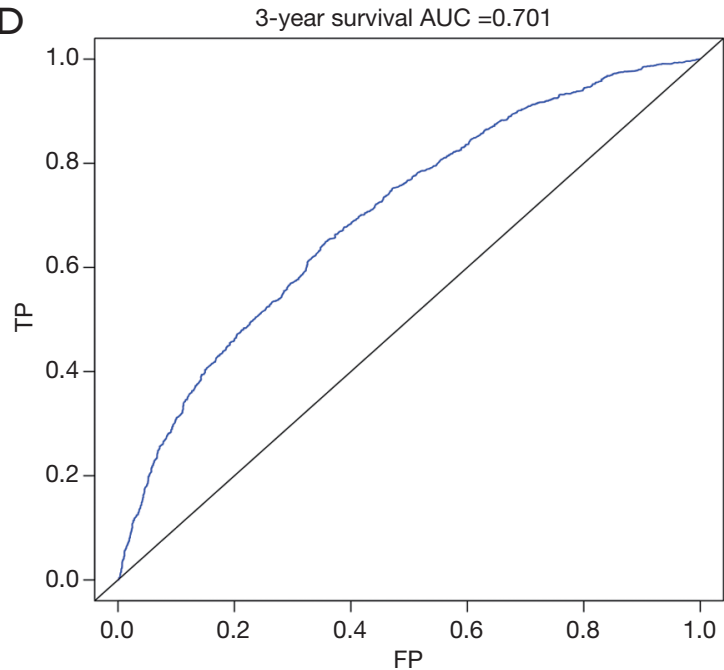

$\mathrm{F}$

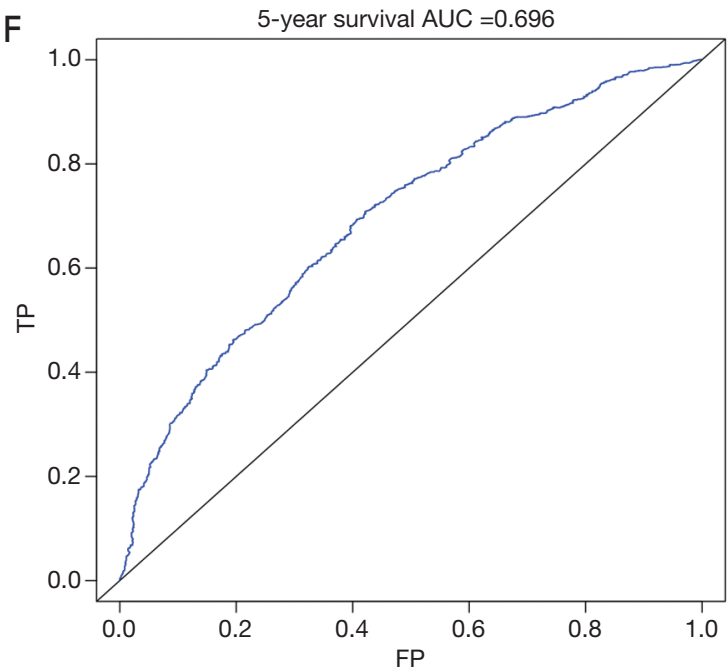

Figure 5 ROC curve of overall survival (OS). ROC curves for 1 year (A), 3 years (C), and 5 years (E), respectively, validated by the model establishment group; ROC curves for 1 year (B), 3 years (D), and 5 years (F), respectively, validated by the validation group. AUC, area under the ROC curve. 
A

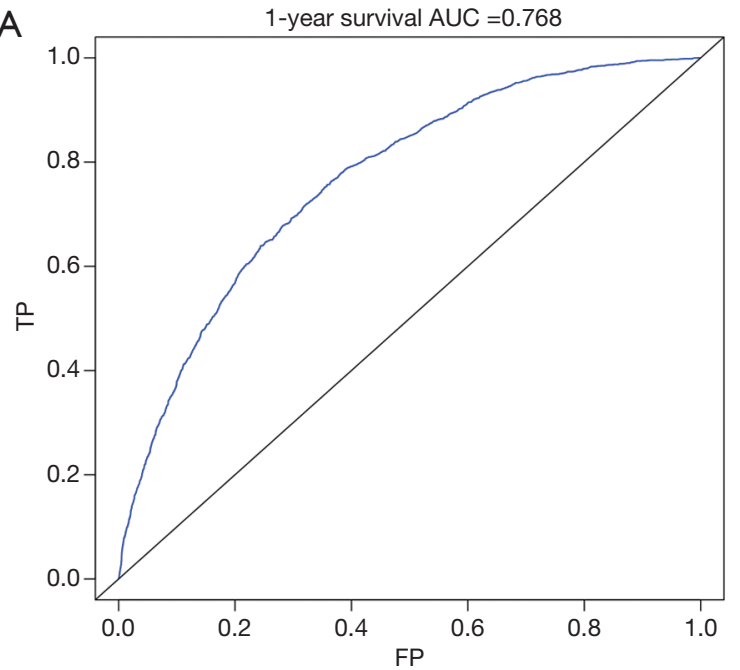

C

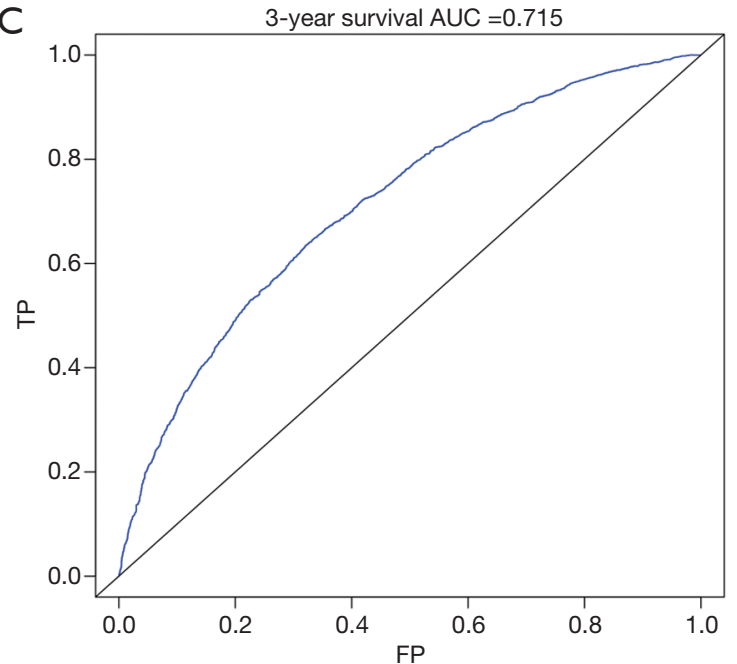

$E$

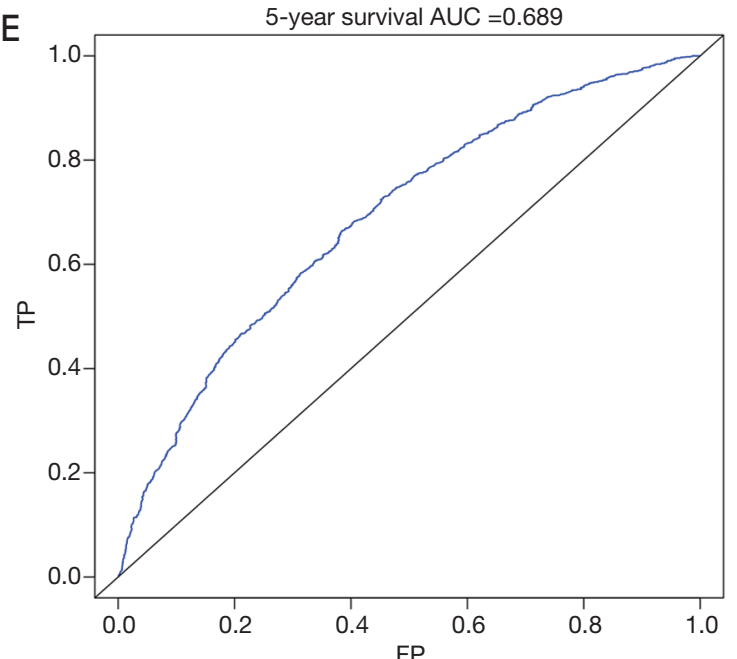

B

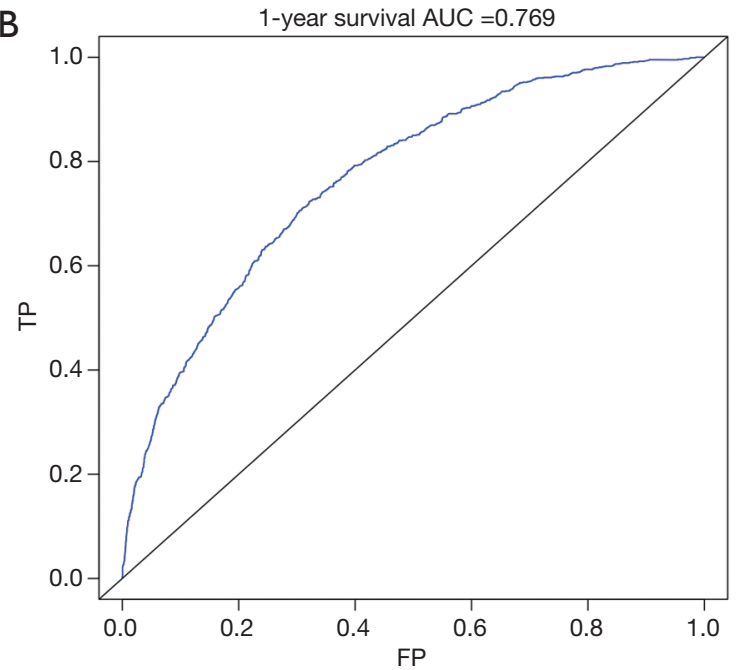

D

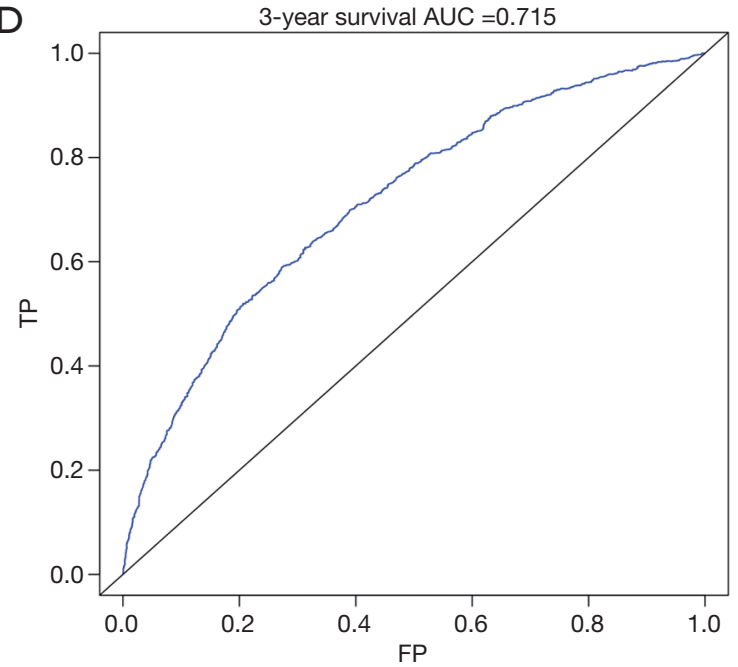

$\mathrm{F}$

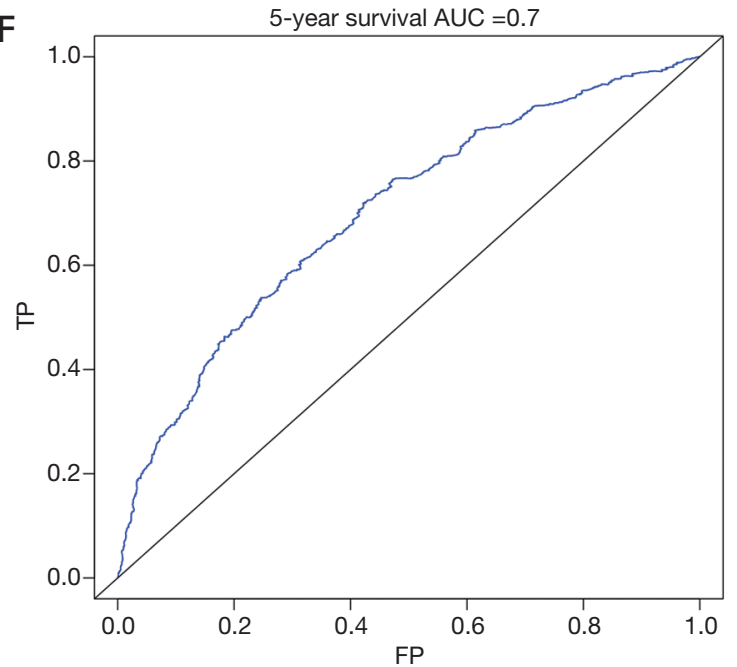

Figure 6 ROC curve of breast cancer-related survival (BCRS). ROC curves for 1 year (A), 3 years (C), and 5 years (E), respectively, validated by the model establishment group; ROC curves for 1 year (B), 3 years (D), and 5 years (F), respectively, validated by the validation group. AUC, area under the ROC curve. 
A

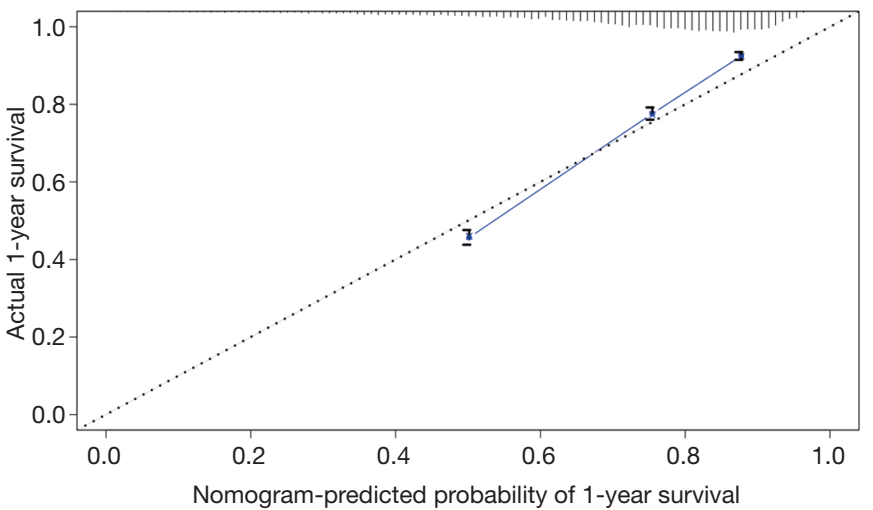

$n=8120 d=4600 p=41,2400$ subjects per group Gray: ideal

C

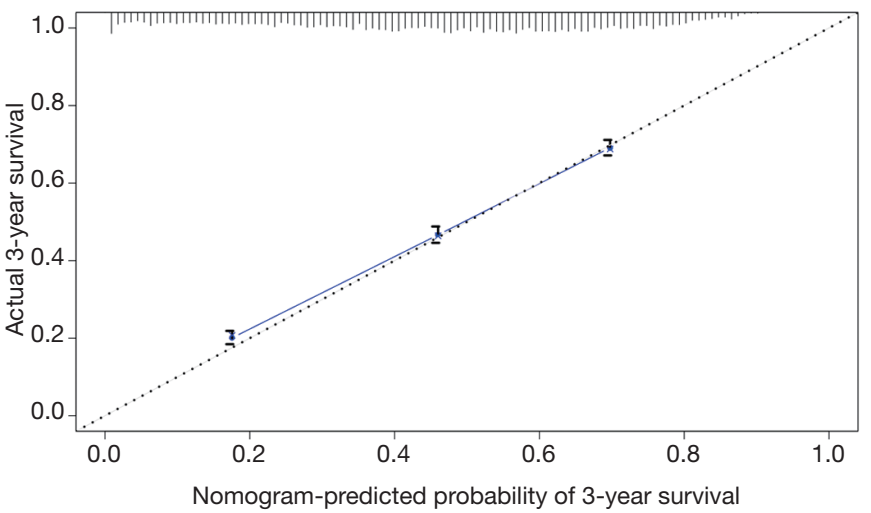

$n=8120 d=4600 p=41,2400$ subjects per group Gray: ideal

X-resampling optimism added, $B=100$ Based on observed-predicted

E

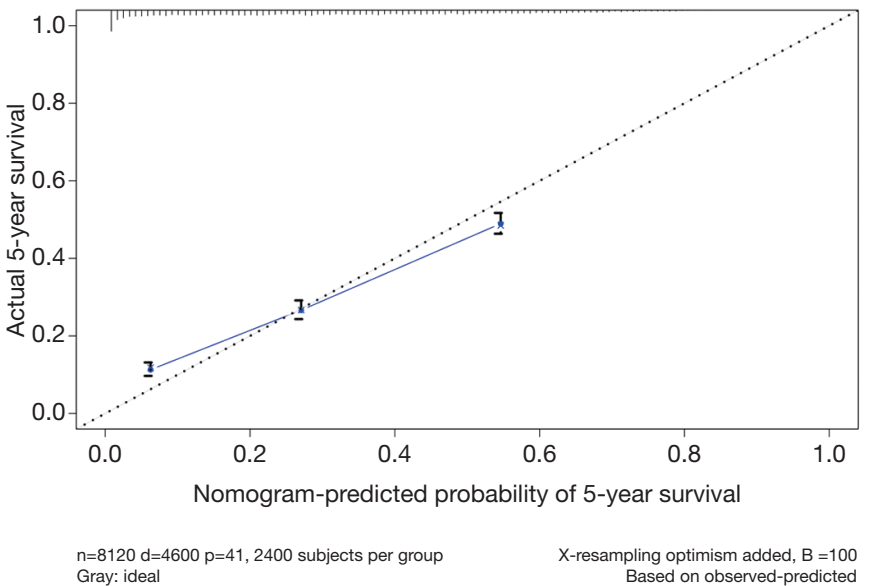

B

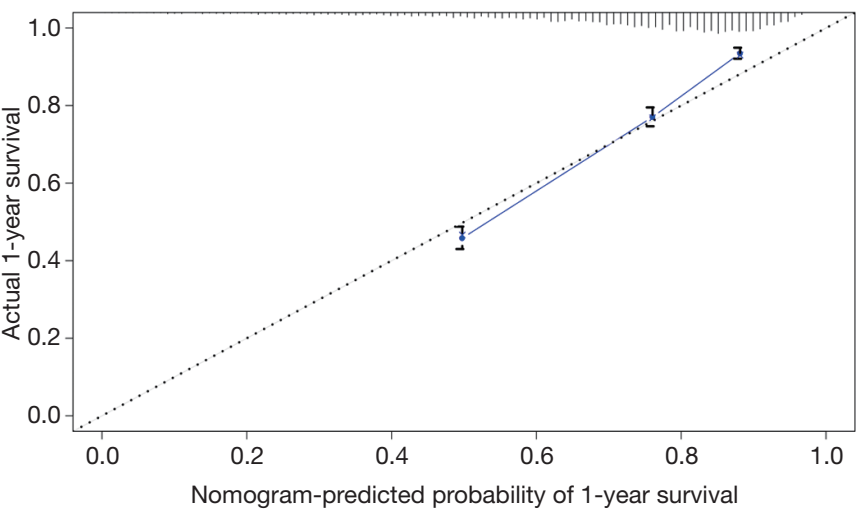

$n=3478 d=1905 p=41,900$ subjects per group Gray: ideal

X-resampling optimism added, $\mathrm{B}=100$ Based on observed-predicted

D

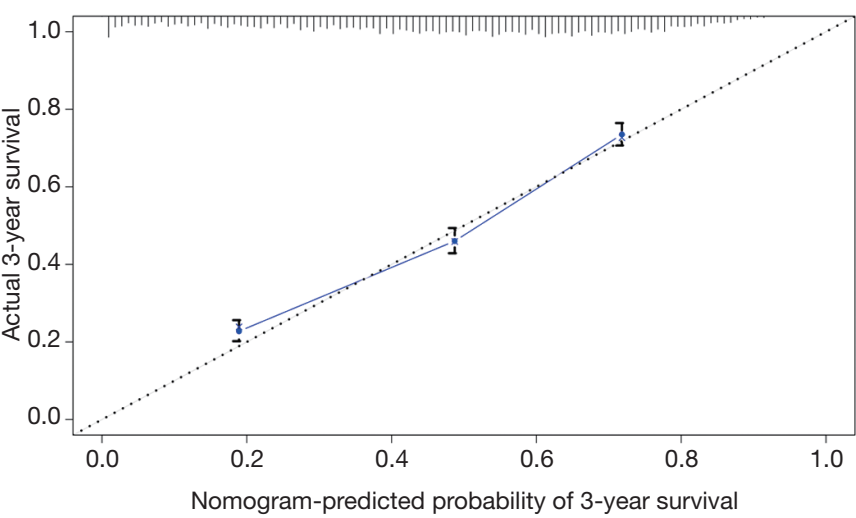

$n=3478 d=1905 p=41,900$ subjects per group $\quad$ X-resampling optimism added, $B=100$ Based on obst

$\mathrm{F}$

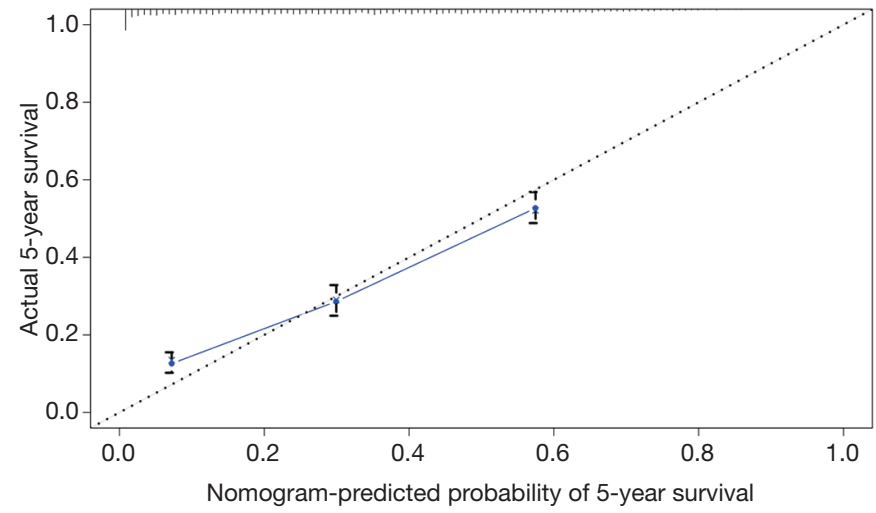

$n=3478 d=1905 p=41,900$ subjects per group X-resampling optimism added, $B=100$ Gray: ideal

Figure 7 Calibration plots of overall survival (OS). Calibration plots for 1 year (A), 3 years (C), and 5 years (E), respectively, validated by the model establishment group. Calibration plots for 1 year (B), 3 years (D), and 5 years (F), respectively, validated by the validation group. 
A

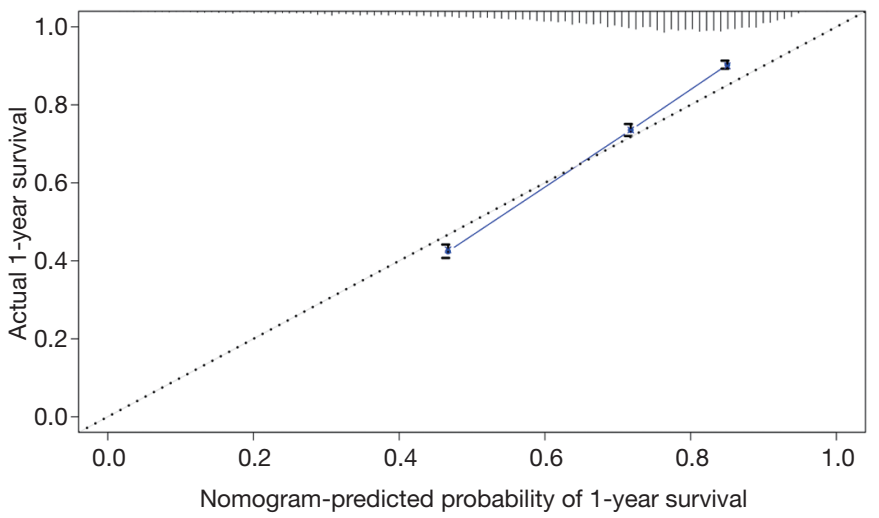

$n=9644 d=6106 p=41,2500$ subjects per group Gray: ideal

C

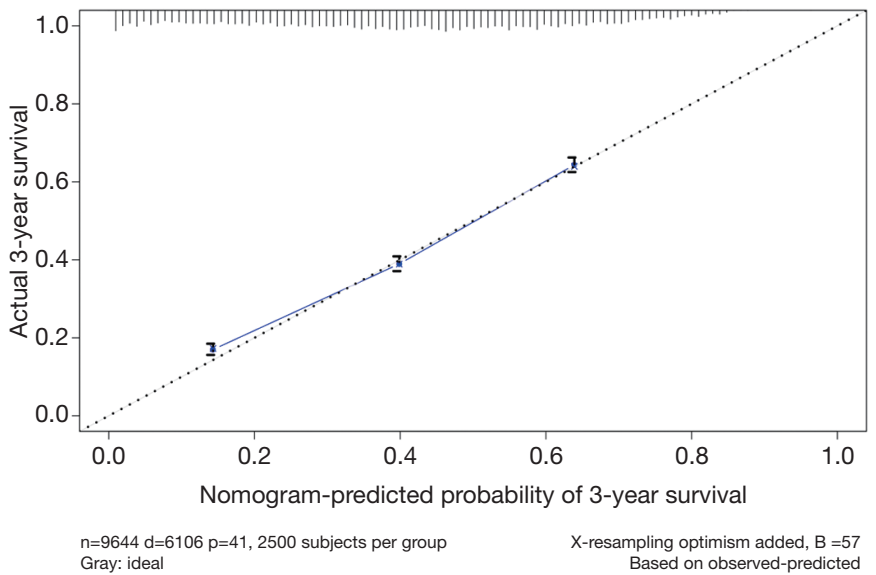

E

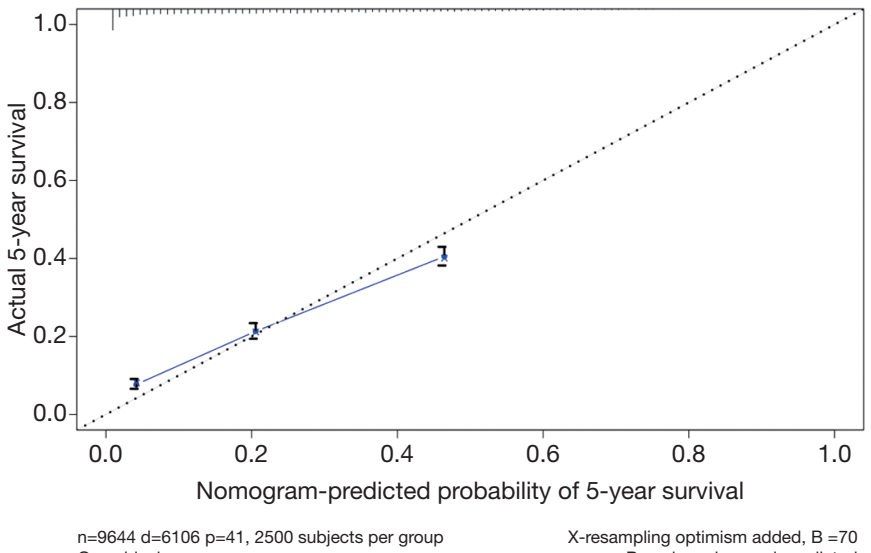

B

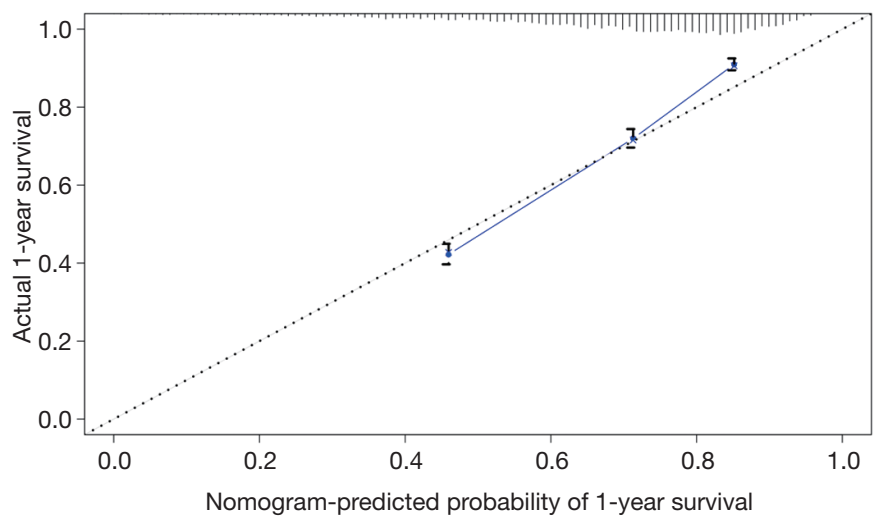

$n=4129 d=2574 p=41,1100$ subjects per group $\quad X$-resampling optimism added, $B=100$ Gray: ideal

Based on obserre

D

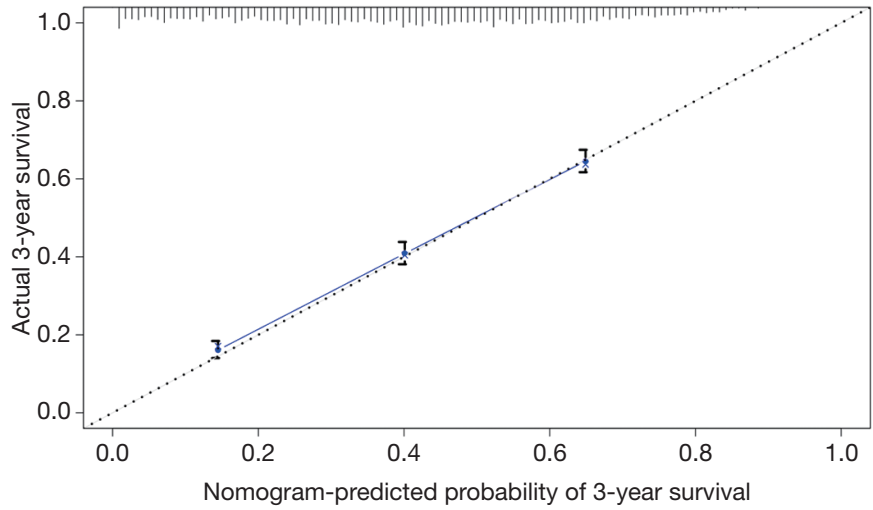

$n=4129 d=2574 p=41,1100$ subjects per group

X-resampling optimism added, $\mathrm{B}=100$ Based on observed-predicted

$\mathrm{F}$

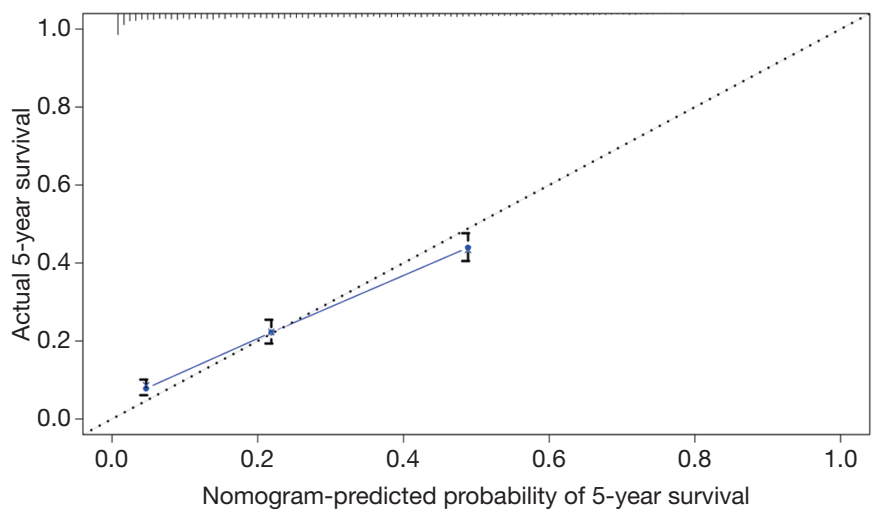

$n=4129 d=2574 p=41,1100$ subjects per group

X-resampling optimism added, $B=100$ Gray: ideal

Figure 8 Calibration plots of breast cancer-related survival (BCRS). Calibration plots for 1 year (A), 3 years (C), and 5 years (E), respectively, validated by the model establishment group. Calibration plots for 1 year (B), 3 years (D), and 5 years (F), respectively, validated by the validation group. 
key to developing an individualized treatment plan is to fully evaluate the prognosis of the patient. The SEER database provides a wealth of complete information on demographics, oncology, and treatment of breast cancer patients, providing appropriate sample data for establishing clinical predictive models.

\section{Demographic information for BCBM patients}

Median survival time is the most intuitive indicator of the prognosis of BCBM patients. Sciubba et al. (15) reviewed 327 patients with bone metastases, and the median survival of the overall cohort was 21.7 months. In our study, 13,773 patients with confirmed breast cancer with bone metastases were included, with a median survival of 20.0 months, similar to previous reports. Our research is more convincing due to the expansion of the sample size. In entire group, the main age of patients was 46-65 years old (48.2\%) and 66-85 years old $(34.7 \%)$, which was consistent with the double-peak pattern of breast cancer in women, the age of onset of early peak was 52 years old, and the age of onset of late peak was 71 years old $(16,17)$. In addition, the increase in age is accompanied by a gradual increase in mortality, so age is considered to be one of the important factors predicting prognosis. In terms of gender, the literature reports that male breast cancer is a relatively rare disease, accounting for about $1 \%$ of breast cancer patients (18). In our study, 173 (1.3\%) male breast cancer patients with bone metastases were included, although the proportion was not high. However, it is still higher than the documented incidence rate. On the one hand, it shows that the incidence of breast cancer in men is low. On the other hand, it is indicated that male breast cancer is generally not easy to attract attention, so it is mostly advanced at the time of diagnosis. However, gender differences did not result in significant differences in BCRM between male and female (56.7\% vs. $56.1 \%, \mathrm{P}=0.876$ ). In terms of ethnicity, 10,612 (77.0\%) white BCBM patients were included in the study, which constitute the main ethnic group in our study. According to the literature, although the incidence of white breast cancer is higher, the mortality rate of black breast cancer patients is higher, which is consistent with the results of our study (19). The breast cancer-related mortality rates of black and white in our study are $63.6 \%$ and $55.2 \%$, respectively. Marital status is considered to be an important factor in the development of breast cancer, and unmarried status is a high-risk factor for breast cancer (20). In our study, the proportion of patients who were unmarried in the study was higher than the married status (51.9\% vs. $42.7 \%)$. In addition, unmarried patients also had higher BCRM than married patients (61.2\% vs. $50.2 \%)$.

\section{Tumor pathology and treatment information for BCBM patients}

In entire group, grade II (34.7\%) and grade III (30.9\%) were dominant. As with other tumors, the degree of differentiation was low, and the mortality rate of patients with high altitude was higher. Among the histological types, ductal carcinoma patients $(61.6 \%)$ had the most, but the BCRM of adenocarcinoma was higher than that of ductal carcinoma and lobular carcinoma $(62.8 \%$ vs. $53.7 \%$ vs. $53.7 \%)$. In recent years, DNA microarray technology and multi-gene RT-PCR quantitative detection methods for molecular classification of breast cancer to predict the risk of breast cancer recurrence and metastasis and its response to treatment, the molecular sub-technical technology combined with immunohistochemistry, breast cancer can be classified into four categories: HR+/HER2- (Luminal A), HR+/HER2 + (Luminal B), HR-/HER2 + (HER2 enriched), and HR-/HER2- (Triple Negative) $(19,20)$. The clinical response and survival of different molecular subtypes of breast cancer are different, and more and more attention has been paid to it. Among the four subtypes, luminal A is the most common, and studies have shown that the percentage of breast cancer in each subtype is $50 \%, 14.1 \%, 12.7 \%$, and $23.2 \%$, respectively. In our study, luminal A still accounted for the vast majority, with the percentages of each subtype being $57.7 \%, 13.4 \%, 5.2 \%$, and $7.8 \%$, respectively. In terms of treatment, it can be clearly observed in Table 3 that patients who did not receive surgery, radiotherapy or chemotherapy had significantly higher BCRM than patients who received the corresponding treatment. This also suggests that aggressive treatment can help improve the prognosis of $\mathrm{BCBM}$ patients.

\section{Evaluation of predictive models}

Prognostic factors with $\mathrm{P}<0.001$ were selected by log-rank test, and nomograms of OS and BCRS were constructed according to multivariate cox regression analysis. Internal and external verification of nomograms using C-index, ROC curves and calibration plots. The $\mathrm{C}$ - index represents the predictive accuracy of nomograms, and the C-index of both nomograms is greater than 0.7 , achieving moderate prediction accuracy. The AUC of the ROC curve represents 
the prediction accuracy of nomograms. For the OS nomogram, only the validation group had an AUC of less than 0.7 in the 5 -year survival prediction, demonstrating that the 1- and 3-year survival prediction models of OS achieved moderate accuracy. For the BCRS nomogram, only the model establishment group had an AUC of less than 0.7 in the 5-year survival prediction, demonstrating that the 1and 3-year survival prediction models of BCRS achieved moderate accuracy. The calibration chart can assess the consistency of the predicted and observed conditions. The 1-, 3- and 5-year calibration plots of OS and BCRS show an excellent consistency, which proves that the two nomograms have good predictive ability. The predictive model of this study has been tested for predictive ability by three methods and has achieved satisfactory results. In addition, the model is based on a large sample of the SEER database and is more convincing.

\section{Limitations}

This study is based on a retrospective study conducted by the SEER database. Due to the limitations of the data included in the database itself, more detailed patient information is not available. We are unable to obtain the patient's physical condition before diagnosis, whether it is accompanied by other diseases, surgical methods, chemotherapy drugs, dose of radiotherapy, and the specific follow-up time for each patient, which limits our further evaluation. In addition, we are unable to obtain short-term or long-term complications after treatment, which severely limits our effective judgment of prognosis. Finally, this study uses only a set of data to split the internal and external verification of the prediction model, which itself has a great bias. However, because the objectivity and authenticity of the SEER database can be guaranteed, we still have reason to believe the nomograms obtained in this study, and then we can further select other samples to verify the model.

\section{Conclusions}

In this study, the SEER database was collected to analyze the factors affecting the prognosis of patients with BCBM, and to select a number of factors that have significant effects on prognosis to establish a predictive model. The final nomograms obtained satisfactory results after a series of internal and external verifications, verifying the accuracy of their predictions. Other samples are needed in the future for more comprehensive external validation of the model, but at this stage, this model will help physicians and patients to have a more accurate judgment of the prognosis.

\section{Acknowledgments}

Thanks to my wife, Mrs Sun, for her support for my life and research.

\section{Footnote}

Reporting Checklist: The author has completed the STROBE reporting checklist. Available at http://dx.doi.org/10.21037/ tbcr-20-14

Conflicts of Interest: The author has completed the ICMJE uniform disclosure form (available at http://dx.doi. org/10.21037/tbcr-20-14). The author has no conflicts of interest to declare.

Ethical Statement: The author is accountable for all aspects of the work in ensuring that questions related to the accuracy or integrity of any part of the work are appropriately investigated and resolved. Ethical Approval and Informed Consent are not applicable. The data comes from the public SEER database. The database has completed Ethical Approval/Informed Consent when acquiring relevant data.

Open Access Statement: This is an Open Access article distributed in accordance with the Creative Commons Attribution-NonCommercial-NoDerivs 4.0 International License (CC BY-NC-ND 4.0), which permits the noncommercial replication and distribution of the article with the strict proviso that no changes or edits are made and the original work is properly cited (including links to both the formal publication through the relevant DOI and the license). See: https://creativecommons.org/licenses/by-nc-nd/4.0/.

\section{References}

1. Bray F, Ferlay J, Soerjomataram I, et al. Global Cancer Statistics 2018: GLOBOCAN Estimates of Incidence and Mortality Worldwide for 36 Cancers in 185 Countries. CA Cancer J Clin 2018;68:394-424.

2. Martin TJ, Moseley JM. Mechanisms in the skeletal complications of breast cancer. Endocr Relat Cancer 2000;7:271-84.

3. Huber S, Ulsperger E, Gomar C, et al. Osseous metastases 
in breast cancer: radiographic monitoring of therapeutic response. Anticancer Res 2002;22:1279-88.

4. Yardley DA. Pharmacologic management of bone-related complications and bone metastases in postmenopausal women with hormone receptor-positive breast cancer. Breast Cancer 2016;8:73-82.

5. George R, Jeba J, Ramkumar G, et al. Interventions for the treatment of metastatic extradural spinal cord compression in adults. Cochrane Database Syst Rev 2015;9:CD006716.

6. Bezuhly M, Temple C, Sigurdson LJ, et al. Immediate postmastectomy reconstruction is associated with improved breast cancer-specific survival: Evidence and new challenges from the Surveillance, Epidemiology, and End Results database. Cancer 2009;115:4648-54.

7. Yang S, Li C, Shi X, et al. Primary Squamous Cell Carcinoma in the Thyroid Gland: A Population-Based Analysis Using the SEER Database. World J Surg 2019;43:1249-55.

8. Harrell FE, Lee KL, Mark DB. Multivariable prognostic models: issues in developing models, evaluating assumptions and adequacy, and measuring and reducing errors. Stat Med 1996;15:361-87.

9. Linden A. Measuring diagnostic and predictive accuracy in disease management: an introduction to receiver operating characteristic (ROC) analysis. J Eval Clin Pract 2006;12:132-9.

10. Heagerty PJ, Zheng Y. Survival Model Predictive Accuracy and ROC Curves. Biometrics 2005;61:92-105.

11. Woolston C. Breast cancer. Nature 2015;527:S101.

doi: $10.21037 /$ tbcr-20-14

Cite this article as: Hua KC. Prognosis prediction model for patients with breast cancer with bone metastasis: based on a population database. Transl Breast Cancer Res 2021;2:3.
12. Okada E, Nakamura M, Koshida Y, et al. Breast carcinoma metastasis to meningioma in the thoracic spine: A case report and review of the literature. J Spinal Cord Med 2015;38:231-5.

13. Schulz M, Lamont D, Muthu T, et al. Metastasis of Breast Cancer to a Lumbar Spinal Nerve Root Ganglion. Spine 2009;34:E735-9.

14. Chan-Seng E, Charissoux M, Larbi A, et al. Spinal Metastases in Breast Cancer: Single Center Experience. World Neurosurg 2014;82:1344-50.

15. Sciubba DM, Gokaslan ZL, Suk I, et al. Positive and negative prognostic variables for patients undergoing spine surgery for metastatic breast disease. Eur Spine J 2007;16:1659-67.

16. López-O'Rourke VJ, Orient-López F, Fontg-Manzano F, et al. Pathological Vertebral Compression Fracture of C3 Due to a Breast Cancer Metastasis in a Male Patient. Spine 2009;34:E586-90.

17. Anderson WF, Althuis MD, Brinton LA, et al. Is Male Breast Cancer Similar or Different than Female Breast Cancer?. Breast Cancer Res Treat 2004;83:77-86.

18. Fentiman IS, Fourquet A, Hortobagyi GN. Male breast cancer. Lancet 2006;367:595-604.

19. Rouzier R, Perou CM, Symmans WF, et al. Breast Cancer Molecular Subtypes Respond Differently to Preoperative Chemotherapy. Clin Cancer Res 2005;11:5678-85.

20. Perou CM. Molecular portraits of human breast tumours. Nature 2000;406:747-52. 